\title{
SINGLE LANGMUIR PROBE \\ CHARACTERISTIC IN A MAGNETIZED \\ PLASMA AT THE TEXT TOKAMAK
}

by

STEFAN JACHMICH

THESIS

Presented to the Faculty of the Graduate School of

The University of Texas at Austin

in Partial Fulfillment

of the Requirements

for the Degree of

MASTER OF ARTS IN PHYSICS

THE UNIVERSITY OF TEXAS AT AUSTIN

May, 1995

\section{DISCLAIMER}

This report was prepared as an account of work sponsored by an agency of the United States Government. Neither the United States Government nor any agency thereof, nor any of their employees, makes any warranty, express or implied, or assumes any legal liability or responsibility for the accuracy, completeness, or usefulness of any information, apparatus, product, or process disclosed, or represents that its use would not infringe privately owned rights. Reference herein to any specific commercial product, process, or service by trade name, trademark, manufacturer, or otherwise does not necessarily constitute or imply its endorsement, recommendation, or favoring by the United States Government or any agency thereof. The views and opinions of authors expressed herein do not necessarily state or reflect those of the United States Government or any agency thereof.

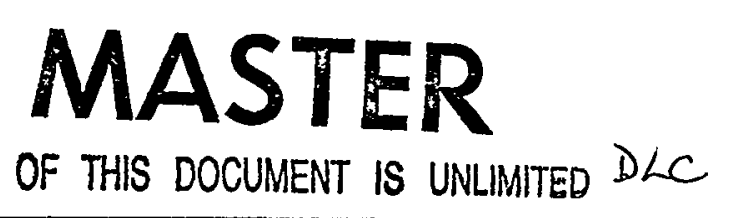




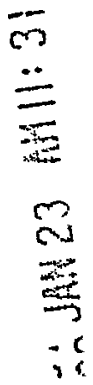




\section{DISCLAIMER}

Portions of this document may be illegible in electronic image products. Images are produced from the best available original document. 
ABSTRACT

\title{
SINGLE LANGMUIR PROBE \\ CHARACTERISTIC IN A MAGNETIZED \\ PLASMA AT THE TEXT TOKAMAK
}

\author{
by \\ Stefan Jachmich \\ Supervising Professor: Roger D. Bengtson
}

A single Langmuir probe tip was used at TEXT-Upgrade to obtain I-V characteristics in a magnetized plasma. Noisy data were reduced by a boxcar-averaging routine.

Unexpected effects, namely non-saturation of ion current, hysterises in the characteristics and $\mathrm{I}(\mathrm{V})$-data were observed, which are in disagreement to the common single probe model. A double probe model allows parameterization of the $\mathrm{I}(\mathrm{V})$ curves and to determine the plasma properties in the scrape-off layer. It is shown in this model that a Langmuir probe does perturb the local space potential in the plasma.

Comparisons were made with the triple probe technique of measuring temperatures. The non-saturation of ion current leads to an error in the triple probe technique of order $20 \%$. 


\section{Contents}

1 Introduction 1

2 Langmuir probe theory 4

2.1 Introduction . . . . . . . . . . . . 4

2.2 Sheath analysis . . . . . . . . . . . . 4

2.3 Particle flux . . . . . . . . . . . . 6

2.3.1 Effect of strong magnetic fields $\ldots \ldots \ldots \ldots 6$

2.3.2 The ion branch . . . . . . . . . . 7

2.3.3 Electron collection . . . . . . . . . . 8

2.3 .4 Summary . . . . . . . . . . . . 12

2.4 Single probe model . . . . . . . . . . . . 13

2.5 Asymmetric double probe model . . . . . . . . . . 19

2.6 Non-saturation effect of the ion current . . . . . . . 25

3 Description of Experimental Apparatus 29

3.1 Langmuir probes at the TEXT-U Tokamak . . . . . . 29

3.2 Power supply and data acquisition system $\ldots \ldots \ldots .30$

3.3 Data analysis . . . . . . . . . . . . . . 31

3.4 Experimental results $\ldots \ldots \ldots \ldots \ldots \ldots \ldots$ 
3.4.1 Comparison of probe models . . . . . . . . 35

3.4 .2 Plasma parameter profiles . . . . . . . . . 36

3.4 .3 Non-saturation effect . . . . . . . . . . 37

4 Conclusion $\quad 50$

viii 


\section{List of Tables}

2.1 $\beta$ values for various gases. The collection areas are assumed

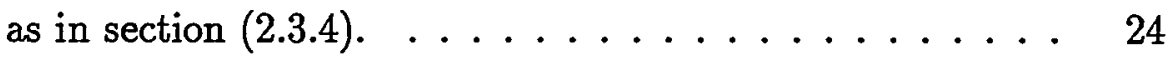

3.1 Comparison of theoretical and experimental values for nonsaturation effect. . . . . . . . . . . . 38 


\section{List of Figures}

1.1 Ratio of known available oil resources over production. Apparently is the rate of discovering new oil reserves higher is than the growth in production (based on data from [1] and

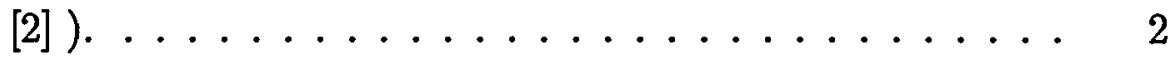

2.1 Plasma potential and density distribution along flux tube. Adapted from $[6] \ldots \ldots \ldots \ldots \ldots$

2.2 Flux tube parallel to the magnetic field. . . . . . . . 10

2.3 Theoretical single probe I-V characteristic. A sharp knee in the curve occurs at the space potential $\ldots \ldots \ldots \ldots$

2.4 Floating Potential distribution in poloidal direction. An active probe causes growth of the space potential. . . . . . 20

2.5 Space potential within the flux tube. The boundaries may not be correct. . . . . . . . . . . . . 21

2.6 Space potential as a function of probe voltage. The plotted curve is based on density and temperature as in shot 205199 (see fig 3.6 ). . . . . . . . . . . . . . . 28

3.1 Schematic of probe head $\ldots \ldots \ldots \ldots \ldots \ldots$ 
3.2 Electrical Circuit . . . . . . . . . . . . .

3.3 Comparison of boxcar-averaging with and without correction for the cable . . . . . . . . . . . . .

3.4 I-V characteristics of several shots at same discharge conditions, but different radial positions. . . . . . . . . . .

3.5 Comparison of data to the corresponding curve of a single probe model as in equation $(2.41) \ldots \ldots \ldots \ldots$

3.6 Curvefit of same data as above based on the asymmetric double probe equation $(2.50) . \ldots \ldots \ldots \ldots$

3.7 First derivative of $\mathrm{I}(\mathrm{V})$ curve of a probe tip positioned $3 \mathrm{~cm}$ behind the limiter. . . . . . . . . . . . . .

3.8 Second deriveative, after applying a cubic spline routine to the first deivative. . . . . . . . . . .

3.9 Logarithm Plot after subtracting the ion-saturation current. Additionally the logarithm plots of the curves as in figure 3.5 and 3.6 are shown. . . . . . . . . . . .

3.10 Density Profiles obtained by curve-fitting using the double probe model and averaging over several shots at same positions respectively. . . . . . . . . . . . 44

3.11 Temperature Profile based on same shots as in figure 3.10 . 45

3.12 Profile of space and floating potential. . . . . . . . 46

3.13 Discharge with probe tip $2 \mathrm{~cm}$ behind the limiter. The solid curve shows the results of a regular asummetric double probe

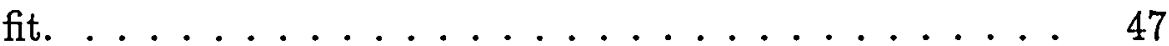

3.14 Data of a symmetric double probe configuration used for turbulence study at TEXT before the upgrade. . . . . . 48 
3.15 Single probe measurements with a large probe tip. The probe was DC-biased superimposed by a AC-voltage. . . . 49 


\section{Chapter 1}

\section{Introduction}

Although it seems, that human society is only focusing on research of new energy providing techniques instead of considering new ways in avoiding energy consumption, nuclear fusion is still a reasonable research field, especially since the knowledge from fusion research has mainly only non-military applications. It is a sophism to declare fusion research as unnecessary, because fuel resources (in any form) grow as technology discovers new methods for oil exploration and production, as could be concluded from figure 1.1. Our society must rather find a more responsible utilization of nature and environment. Otherwise - due to the fact of natural limit of all energy resources - humanity will end up at some time in burning itself up for energy production.

Fusion, as a major research field with a goal of safe and economic energy production, has for its main task plasma confinement and understanding the resulting instabilities. In order to ignitiate the fusion process, a completely ionized gas ("plasma") has to be confined magnetically at a 


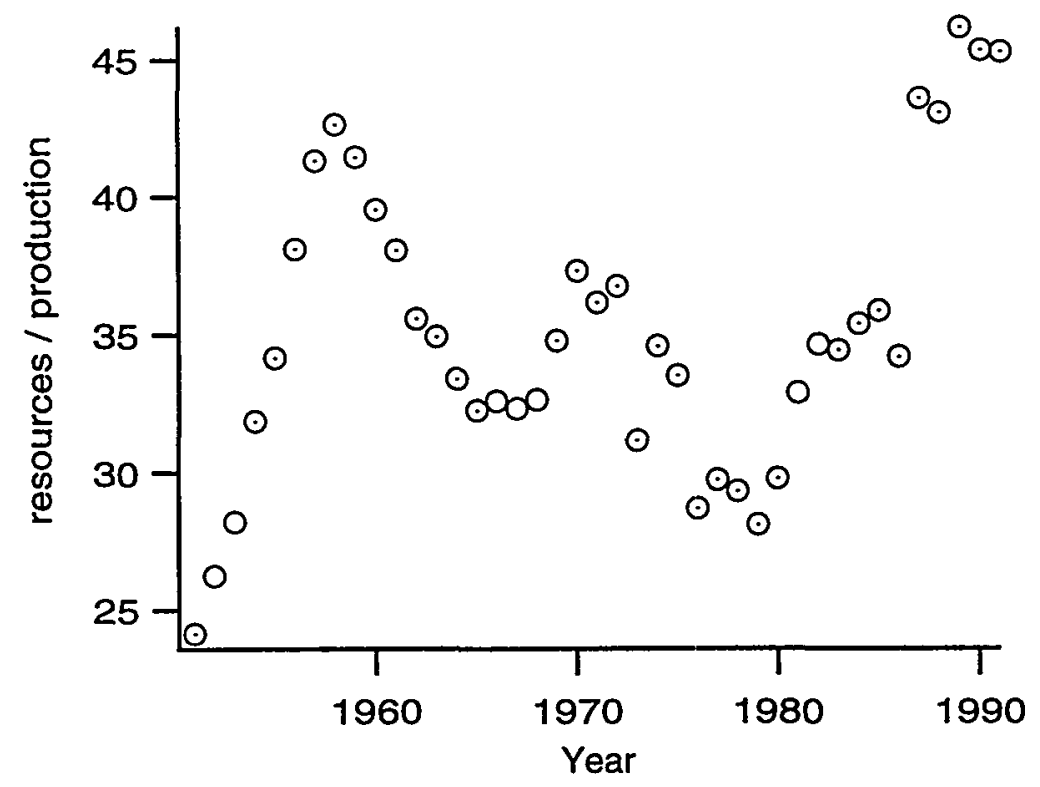

Figure 1.1: Ratio of known available oil resources over production. Apparently is the rate of discovering new oil reserves higher is than the growth in production (based on data from [1] and [2]).

certain density and temperature for a long enough time. A magnetic field configuration, which is very promising to constrain plasma for sustainable energy production, is a device called Tokamak. The first Tokamak was built in 1955 in the former Soviet Union. A high current (at TEXT $I_{P} \sim 200 \mathrm{kA}$ ) is driven through the plasma. The resulting magnetic field restrains the plasma, in alliance with an external toroidal magnetic field, from reaching the wall. In addition limiters (s.fig 3.2) are usually installed to protect the wall from charged particles. The outer plasma region, behind the limiters is the scrape-off layer plasma (SOL).

Since the plasma density and temperature in the SOL are a factor of ten smaller than in the center, electrostatic probes can be used for 
plasma property measurements. Irving Langmuir (1881 - 1957) pioneered their use as an experimentally simple diagnostic. However the simplicity of Langmuir probes makes us pay with a rather complicated theory. Even this project, originally planned for 8 months, suffered from this "fundamental law of plasma physics".

Applying a voltage to the probe and measuring the corresponding current originates the typical $I(V)$ curves. The originally object of this project was to record several characteristics at different radial position by sweeping a AC-voltage at particular frequencies. Due to the frequency limit of the power supply, only a frequency range up to $200 \mathrm{kHz}$ was available, in which no significant changes were observed. In addition other aspects of I(V)-curves were discovered, namely hysterises effects, which cannot be completely attributed to the power supply, and a current-voltage dependence, which definitely does not follow the common single probe model.

In the following chapter the theoretical basic of two different probe models is introduced. After a short description of the experimental apparatus in chapter 3, various mathematical methods are applied to experimental results in order to determine plasma potential, density, temperature and floating potential. 


\section{Chapter 2}

\section{Langmuir probe theory}

\subsection{Introduction}

Electrical probes are in general helpful for local particle flux measurements. Langmuir and Mott-Smith [3] developed the use and theory of so-called Langmuir probes seventy years ago. Important work in this field was also done by Chen [4] and Laframboise [5]. Later Stangeby [6] and Hutchinson [7] extended the fluid model to enable a more accurate estimate of the particle flux. In this chapter various probe models and their accuracy will be discussed.

\subsection{Sheath analysis}

For simplification the plasma is assumed to be collisionless and nonmagnetized. Corrections for the case of a magnetized plasma are shown later.

When any electrical conductor, held at a potential $V_{p r}$, different 
from the existing undisturbed space potential $V_{s p}$, is inserted into the plasma, a sheath builds up around the conductor. The potential drop is over a shorter range in a plasma than in vacuum, since the charged particles try to redistribute themselves so that the plasma is shielded from the electric field generated from the probe. An important parameter in all considerations regarding electric fields is the Debye length $\lambda_{D}$, the penetration length for an electric field in a plasma. Assuming $M$ particle species, having different thermal equilibrium velocity distributions, and $K$ particle species at rest, whereby the charge of each species is not necessary the same, the densities are then

$$
\begin{aligned}
n_{m}(\vec{x}) & =n_{\infty, m} \exp \left(-\frac{q_{m} \Delta V(\vec{x})}{T_{m}}\right) \\
n_{k}(\vec{x}) & =n_{\infty, k}
\end{aligned}
$$

with $\Delta V(\vec{x})=V(\vec{x})-V_{s p}$ and $n_{\infty}$ as the density far from the perturbing charge, where the space potential is undisturbed. Note, that $T_{m}$ is the temperature in energy units. In order to calculate the Debye length $\lambda_{\mathcal{D}}$ we need Poisson's equation

$$
\begin{aligned}
\nabla^{2}\left(\Delta V(\vec{x})+V_{s p}\right) & =-\frac{1}{\epsilon_{0}}\left[\sum_{m}^{M} q_{m} n_{m}(\vec{x})+\sum_{k}^{K} q_{k} n_{k}(\vec{x})\right] \\
\nabla^{2}(\Delta V(\vec{x})) & =-\frac{1}{\epsilon_{0}}\left[\sum_{m}^{M} q_{m} n_{\infty, m} \exp \left(-\frac{q_{m} \Delta V(\vec{x})}{T_{m}}\right)+\sum_{k}^{K} q_{k} n_{\infty, k}\right] .
\end{aligned}
$$

The exponential terms can be expressed by a Taylor series expansion

$$
\exp \left(\frac{-q_{m} \Delta V(\vec{x})}{T_{m}}\right)=1+\frac{-q_{m} \Delta V(\vec{x})}{T_{m}}+\frac{1}{2}\left(\frac{q_{m} \Delta V(\vec{x})}{T_{m}}\right)^{2}+\ldots
$$

For $\left|q_{m} \Delta V(\vec{x})\right| \ll T_{m}$ terms of higher order can be neglected and equation 
(2.3) is linearized to

$\nabla^{2}(\Delta V(\vec{x}))-\frac{1}{\epsilon_{0}} \sum_{m}^{M} q_{m} n_{\infty, m} \frac{q_{m} \Delta V(\vec{x})}{T_{m}}=-\frac{1}{\epsilon_{0}}\left[\sum_{m}^{M} q_{m} n_{\infty, m}+\sum_{k}^{K} q_{k} n_{\infty, k}\right]$.

or, introducing the Debye length $\lambda_{D}$,

$$
\nabla^{2}(\Delta V(\vec{x}))-\frac{1}{\lambda_{D}^{2}} \Delta V(\vec{x})=-\frac{1}{\epsilon_{0}}\left[\sum_{m}^{M} q_{m} n_{\infty, m}+\sum_{k}^{K} q_{k} n_{\infty, k}\right] .
$$

A solution for this differential equation is

$$
\Delta V(\vec{x})=\exp \left(\frac{\vec{x}}{\lambda_{D}}\right)+\frac{\lambda_{D}}{\epsilon_{0}}\left[\sum_{m}^{M} q_{m} n_{\infty, m}+\sum_{k}^{K} q_{k} n_{\infty, k}\right],
$$

whereby $\lambda_{D}$ is defined as

$$
\lambda_{D} \equiv \sqrt{\left(\sum_{m}^{M} \frac{q_{m}^{2} n_{\infty, m}}{\epsilon_{0} T_{m}}\right)^{-1}},
$$

or in the case of electrons and one ion species, Maxwellian distributed at same temperature $T_{e}$,

$$
\lambda_{D}=\frac{\sqrt{\epsilon_{0} T_{e}}}{e \sqrt{n_{\infty, e}+n_{\infty, i}}} .
$$

It should be pointed out, that the Debye length $\lambda_{D}$ does not depend on the particles at rest (see eq 2.2).

\subsection{Particle flux}

\subsubsection{Effect of strong magnetic fields}

We can classify a magnetic field as strong if the Larmor radius of charged particles is substantial smaller than the probe radius

$$
r_{L}=\frac{m v_{\perp}}{q B}<r_{p r}
$$


The electron larmor radius is usually small for most Tokamaks and for TEXT. On the other hand the ion Larmor radius is of comparable size to the probe used for this project.

\subsubsection{The ion branch}

Ions may have a low temperature compared to the electrons in the scrape-off layer. Since the ions may not be in a thermal equilibrium a Maxwellian velocity distribution can't be assumed.

Stangeby developed in 1984 [8] a model for probes in a magnetized plasma, which was later (1987) improved by Hutchinson ([9] - [11]). The main hypothesis was perpendicular ion diffusion into the magnetic flux tube. The low temperature ions are then accelerated up to the speed of sound $c_{s}$. His final result is an ion flux to a positive particle collecting probe, as

$$
\Gamma_{i}=\frac{1}{2} n c_{s}
$$

However a cold ion temperature is not realistic. Hutchinson corrected this by treating the ions with a finite temperature, which causes a non-zero viscosity. The main difference in the results arises from finite velocity flow in the main plasma. The calculations are straight forward and the ion flux is

$$
\Gamma_{i}=0.35 n c_{s} .
$$

Since Hutchinson allows ions to have a finite temperature, he obtains a different effective value for the viscosity (instead of Stangeby, where $\eta \approx 0$ ). If ions have a charge of $Z e$, the speed of sound $c_{s}$ is then defined 
as

$$
c_{s}=\sqrt{\frac{T_{i}+Z T_{e}}{m_{i}}} .
$$

The distinction between a magnetic sheath and the Debye sheath should be made. The magnetic sheath is defined where the ion speed parallel to the magnetic field is $c_{s}$, the Debye sheath, where ions are accelerated up to $c_{s}$, so that the velocity perpendicular to the collection area has reached $c_{s}$. Due to adiabatic expansion the ion temperature cools down at the sheath edge to

$$
T_{i, D}=\left(\frac{n_{i, D}}{n_{i, m}}\right)^{2} T_{i, m}
$$

The sound speed is then

$$
c_{s}=\sqrt{\frac{\gamma T_{i}+Z T_{e}}{m_{i}}},
$$

whereby $\gamma=3$ for adiabatic expansion, if $T_{i} \approx T_{e}$ (for hydrogen: $c_{s}=$ $\left.2 \sqrt{\frac{T_{e}}{m_{i}}}\right)$.

\subsubsection{Electron collection}

Many problems are involved in contemplation of the electron collection. In the following a model, first published by Stangeby [6], is extended to find an expression for electron saturation current.

For attracting potentials a potential distribution as in figure 2.1 is generated. The velocity of electrons is Maxwellian distributed (MVD) for repelling potentials. This is assumed to be valid even for potentials, where electron saturation is reached (curve E), although the thermal equilibrium (as a prerequisite for a MVD) is not fulfilled. However, in order to guarantee continuity in the electron current at the space potential, it is reasonable 
to retain a MVD for all probe potentials, except for the case of probes in a strong magnetic field. A correction for strong magnetic fields is shown in the following section.

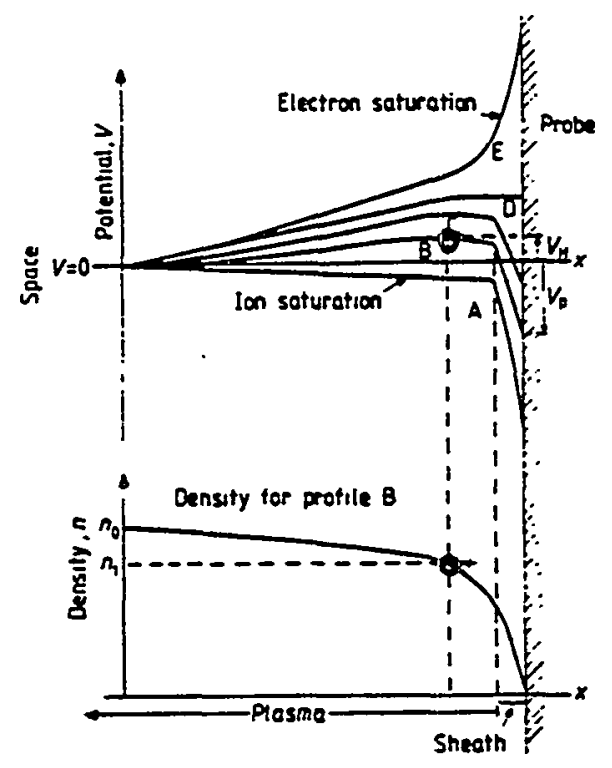

Figure 2.1: Plasma potential and density distribution along flux tube. Adapted from [6]

Because of the magnetic field a flux tube connecting the probe tip with the reference electrode is built up parallel to the magnetic field (fig 2.2). An electric field is necessary to drive current against collisional drag. Moreover the ions are repelled, which reduces their density by the Boltzmann factor. Because of the balance of the penetrated electric field and the quasi neutrality in the presheath the probe current is then caused by parallel electron flux

$$
\Gamma_{\|}=D_{\|}(1+\tau) \frac{d n(x)}{d x},
$$




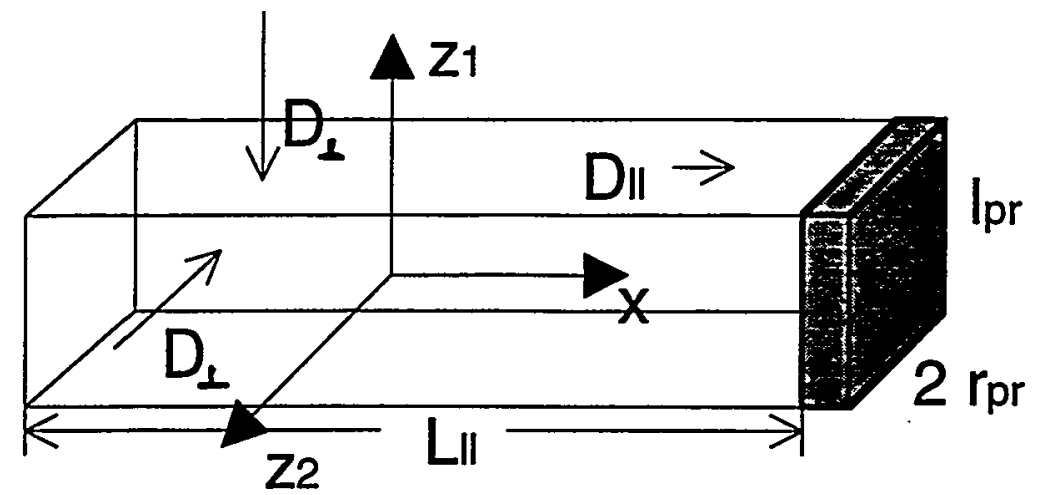

Figure 2.2: Flux tube parallel to the magnetic field.

which is balanced by perpendicular diffusion into the flux tube,

$$
\begin{aligned}
& \Gamma_{\perp, 1}=D_{\perp}(1+\tau) \frac{d n}{d z_{1}}(x) \\
& \Gamma_{\perp, 2}=D_{\perp}(1+\tau) \frac{d n}{d z_{2}}(x)
\end{aligned}
$$

(for a rectangular cross-section),

$$
\Gamma_{\|} A_{\|}=-\left(\Gamma_{\perp, 1} A_{1}+\Gamma_{\perp, 2} A_{2}\right)
$$

The derivatives are approximated by

$$
\begin{aligned}
& \frac{d n(x)}{d x} \approx-\frac{n_{\infty}-n_{c}}{L_{\|}} \\
& \frac{d n}{d z_{1}}(x) \approx \frac{n_{\infty}-n(x)}{l_{p r}} \\
& \frac{d n}{d z_{2}}(x) \approx \frac{n_{\infty}-n(x)}{d_{p r}},
\end{aligned}
$$

with $n(x)=n_{\infty}-\frac{n_{\infty}-n_{c}}{L_{\|}} x$. The length $L_{\|}$is the distance between the crest of the density profile along the $\mathrm{x}$-axis and the point were the density 
$n(x)$ achieves $n_{\infty}$. This characteristic length is not necessary equal to the connection length. The connection length is the length of the flux tube from the probe to a material limiter in the SOL.

After inserting equations (2.17) - (2.19) and integrating over the rhs in equation (2.16), the parallel diffusion can be written as

$$
D_{\|}=D_{\perp} \frac{1}{2}\left(2 \frac{d_{p r}}{l_{p r}} L_{\|}+2 \frac{l_{p r}}{d_{p r}} L_{\|}\right) \frac{L_{\|}}{l_{p r} d_{p r}}
$$

$L_{\|}$can be expressed by the ratio $\kappa \equiv \frac{D_{\perp}}{D_{\|}}$

$$
L_{\|}=\frac{1}{\sqrt{\kappa}} \frac{l_{p r} d_{p r}}{\sqrt{l_{p r}^{2}+d_{p r}^{2}}} .
$$

Replacing the above expression in (2.17) and that in (2.12) gives

$$
\Gamma_{\|}=D_{\|}(1+\tau) \frac{n_{\infty}-n_{c}}{l_{p r} d_{p r}} \sqrt{\kappa} \sqrt{l_{p r}^{2}+d_{p r}^{2}} .
$$

At the crest of the potential profile (where $n \equiv n_{c}$ ) the parallel flux is given by

$$
\Gamma_{\|}=\frac{1}{4} n_{c} v_{t h} .
$$

By inserting this for $n_{c}$ and after rearranging equation (2.21) the corrected term for the electron flux to an attracting probe is received

$$
\begin{aligned}
\Gamma_{e} & =\frac{1}{4} n_{\infty} v_{t h} \frac{\sqrt{\kappa} \sqrt{l_{p r}^{2}+d_{p r}^{2}}(1+\tau) \lambda}{\sqrt{\kappa} \sqrt{l_{p r}^{2}+d_{p r}^{2}}(1+\tau) \lambda+l_{p r} d_{p r}} \\
& =\frac{1}{4} n_{\infty} v_{t h} \frac{\xi}{\xi+1}
\end{aligned}
$$

whereby $\lambda$ is the mean free path and $\xi$ is a correction factor depending on the magnetic field through the perpendicular diffusion coefficient $D_{\perp}$

$$
\xi \equiv \sqrt{\kappa} \sqrt{\frac{1}{l_{p r}^{2}}+\frac{1}{d_{p r}^{2}}}(1+\tau) \lambda=(1+\tau) \frac{\lambda}{L_{\|}} .
$$


The perpendicular diffusion coefficient is small in strong magnetic fields, so that $\xi$ becomes small and

$$
\Gamma_{e}=\frac{1}{4} n_{\infty} v_{t h} \xi
$$

For repelling potentials $\left(V_{p r}<V_{s p}\right)$ the electron must have a certain energy to get over the potential wall. Integrating over a one-dimensional velocity distribution $f_{x}\left(v_{x}\right) d v_{x}=n_{0} \sqrt{\frac{m_{e}}{2 \pi T_{e}}} \exp \left(-\frac{m_{e} v_{x}}{2 T_{e}}\right)$ gives the electron current

$$
\begin{aligned}
I_{e}\left(V_{p r}\right) & =-A_{c, e} e n_{\infty} \int_{v_{x 0}}^{\infty} v_{x} \exp \left(-\frac{m_{e}}{2 T_{e}} v_{x}^{2}\right) d v_{x} \\
& =-\frac{1}{4} A_{c, e} e n_{\infty} \sqrt{\frac{8 T_{e}}{\pi m_{e}}} \exp \left(\frac{e\left(V_{p r}-V_{s p}\right)}{T_{e}}\right)
\end{aligned}
$$

$\left(v_{x 0}=\sqrt{\frac{2 e}{m_{e}}\left(V_{p r}-V_{s p}\right)}\right)$, and thus the current at the space potential is defined as the electron saturation current

$$
I_{e s}=-\frac{1}{4} A_{c, e} e n_{\infty} v_{t h} \xi
$$

with $v_{t h} \equiv \sqrt{\frac{8 T_{e}}{\pi m_{e}}}$ and the magnetic field correction $\xi$.

\subsubsection{Summary}

The ion flux can be described for probe potential less than the space potential in a hydrogen environment as

$$
\Gamma_{i}=0.7 n_{\infty} \sqrt{\frac{T_{e}}{m_{i}}}
$$

and the ion saturation current $\left(I_{i s}=A_{c, i} e \Gamma_{i}\right)$ as

$$
I_{i s}=1.4 \pi r_{p r} l_{p r} e n_{\infty} \sqrt{\frac{T_{e}}{m_{i}}} .
$$

The electron current is for $V_{p r}<V_{s p}$ 


$$
\begin{aligned}
& \Gamma_{e}=0.25 n_{\infty} \sqrt{\frac{8}{\pi}} \sqrt{\frac{T_{e}}{m_{e}}} \\
& I_{e s}=-\sqrt{\frac{8}{\pi}} r_{p r} l_{p r} e n_{\infty} \sqrt{\frac{T_{e}}{m_{e}}} .
\end{aligned}
$$

We assume a collection area for electrons to be $A_{c, e}=4 r_{p r} l_{p r}$ and for ions $A_{c, i}=2 \pi r_{p r} l_{p r}$. However latter equation should be used only if the ion gyroradius $r_{L}$ is comparable to the size of the probe, as it is in strong magnetic fields.

Again it should be pointed out, that both equations presupposed to know the ion temperature or at least a limit. The equations can vary by factor of two depending on the chosen presumption of cold ions or an ion temperature of order of electron temperature.

\subsection{Single probe model}

A probe configuration is called a single probe if a probe (in this experiment a cylinder) is inserted into the plasma, where the collection area is small compared to the reference electrode (namely the limiters or vacuum vessel). The ion current density for each species $i$ is

$$
J_{i}=Z_{i} e \Gamma_{i}
$$

multiplied with the corresponding collecting area $A_{c, i}$ (which may be different for each species) to give the so-called ion saturation current

$$
I_{i s}=\sum_{i} A_{c, i} Z_{i} e \Gamma_{i}
$$


For retarding probe potential $\left(V_{p r}<V_{s p}\right)$, the electron flux to the probe is reduced by the Boltzmann-factor. Thus the current density is

$$
J_{e}=-e \Gamma_{e} \exp \left(\frac{e \Delta V}{T_{e}}\right),
$$

with $\left(\Delta V \equiv V_{p r}-V_{s p}\right)$ and $e$ as elemental charge.

For a single dominant ion species (for hydrogen $Z_{i}=1$ ) the total probe current is

$$
\begin{aligned}
& I_{p r}=-I_{i s}-I_{e s} \exp \left(\frac{e \Delta V}{T_{e}}\right) \\
& I_{p r}=4 r_{p r} l_{p r} e n_{\infty} \sqrt{T_{e}}\left[-\frac{0.7}{\sqrt{m_{i}}}+\frac{1}{\sqrt{2 \pi m_{e}}} \exp \left(\frac{e \Delta V}{T_{e}}\right)\right] .
\end{aligned}
$$

The point where the current goes to zero is defined as floating potential. The same value can be measured by using an additional unbiased probe tip. Setting equation (2.34) to zero and rearranging produces the well known relation

$$
V_{s p}=V_{f l}+\frac{T_{\mathrm{e}}}{e} \ln \left(-\frac{I_{e s}}{I_{i s}}\right)
$$

or introducing $\gamma \equiv \ln \left(-\frac{I_{e s}}{I_{i s}}\right)$, which is usually of order $2-3$,

$$
V_{s p}=V_{f l}+\gamma \frac{T_{e}}{e} .
$$

Inserting equation (2.36) for $V_{s p}$ in (2.34) gives

$$
I_{p r}=-I_{i s}-I_{e s} \exp \left[\frac{e}{T_{e}}\left(V-V_{f l}-\frac{T_{e}}{e} \ln \left(-\frac{I_{e s}}{I_{i s}}\right)\right)\right],
$$

which leads to a different way to describe $I_{p r}\left(V_{p r}\right)$ for $\Delta V<0$

$$
I_{p r}=-I_{i s}\left[1-\exp \left(\frac{e\left(V_{p r}-V_{f l}\right)}{T_{e}}\right)\right]
$$

or 


$$
I_{p r}=I_{i s} \frac{1-\exp \left(-\frac{e\left(V_{p r}-V_{f l}\right)}{T_{e}}\right)}{\exp \left(-\frac{e\left(V_{p r}-V_{f l}\right)}{T_{e}}\right)} .
$$

A problem arises to describe the voltage dependence of the probe current for probe potential larger than the space potential. The probe is now attracting electrons and consequently repelling ions. However an assumption for the velocity distribution of the ions has to be made. In the Hutchinson model [9] (in accordance with $T_{i} \approx 0$ ) the ions are accelerated to the speed of sound $c_{s}$, which corresponds to a mono-energetic distribution. Since no electric field $\vec{E}_{x}$ exists for probe potentials at space potential, ions are no longer accelerated and consequently not collected any more. This would lead to a probe characteristic

$$
\begin{array}{ll}
V_{p r}<V_{s p}: & I_{p r}=-I_{i s}-I_{e s} \exp \left(\frac{e \Delta V}{T_{e}}\right) \\
V_{p r}>V_{s p}: & I_{p r}=-I_{i s}-I_{e s} .
\end{array}
$$

But in reality this is not a good approach. Unpublished ion temperature measurements using far line wings of $\mathrm{H}_{\alpha}$ (as in [12]) suggest an ion temperature up to 1.5 - 2 times electron temperature. Than the Hutchinson model cannot be applied and a Maxwellian distribution must be considered for the ions. An ion particle flux, reduced by a Boltzmann-factor, $J_{i}=-e \Gamma_{i} \exp \left(-e \Delta V / T_{e}\right)$

$$
\begin{array}{ll}
V_{p r}<V_{s p}: & I_{p r}=-I_{i s}-I_{e s} \exp \left(\frac{e \Delta V}{T_{e}}\right) \\
V_{p r}>V_{s p}: & I_{p r}=-I_{i s} \exp \left(-\frac{e \Delta V}{T_{e}}\right)-I_{e s}
\end{array}
$$

(for $T_{i} \approx T_{e}$ ) gives certainly a better impression (fig 2.3 ), but is still not satisfying, since a discontinuity in the first derivative still exists at the space 


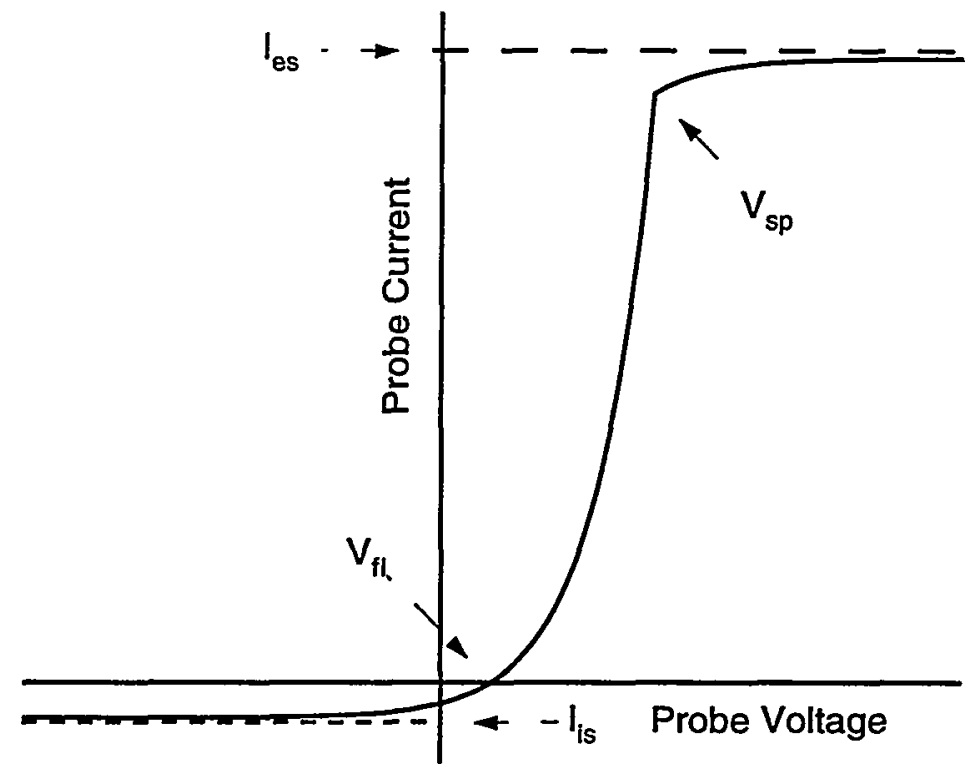

Figure 2.3: Theoretical single probe I-V characteristic. A sharp knee in the curve occurs at the space potential

potential (which does not agree with the experimental data). Nevertheless Laframbois [13] and Sanmartin [14] were able to explain this blurring of the knee.

An accurate determination of the ion temperature is important to estimate the plasma density. Since electron saturation is still not understood and even the ion saturation current formula has some uncertainty, plasma density measurements using Langmuir probes can give only estimates for the density in the scrape-off layer.

The space potential cannot be treated as a free parameter. A reasonable value must be chosen before the remaining plasma parameters can be estimated by a curve fit. Several possibilities using the I-V curve and 
its derivatives give a good estimate for $V_{s p}$.

Under the assumption of any arbitrary electron velocity distribution (EVD) $f\left(v_{x}, v_{y}, v_{z}\right)$ the electron current is for repelling potentials

$$
I_{e}=-A_{c, e} e \int_{-\infty}^{\infty} \int_{-\infty}^{\infty} \int_{\frac{\varepsilon \Delta V}{T_{e}}}^{\infty} v_{x} f\left(v_{x}, v_{y}, v_{z}\right) d v_{x} d v_{y} d v_{z}
$$

Most EVD's are usually a product of three single velocity distributions $f\left(v_{x}, v_{y}, v_{z}\right)=f_{x}\left(v_{x}\right) f_{y}\left(v_{y}\right) f_{z}\left(v_{z}\right)$. After carrying out the integration in two dimensions, equation (2.42), reduces to

$$
I_{e}=-A_{c, e} e \int_{\frac{e \Delta V}{T_{e}}}^{\infty} v_{x} f_{x}\left(v_{x}\right) d v_{x} .
$$

Replacing $v_{x}$ using the law of energy conservation $-E=\frac{1}{2} m v_{x}^{2}-e \Delta V-$, gives

$$
I_{e}=-A_{c, e} e \int_{-e \Delta V}^{\infty}(E+e \Delta V) \dot{f}(E) d E
$$

$\dot{f}$ is the normalized energy distribution function, note that $f \neq f_{x}$. Inserting this into (2.34) and differentiating once with respect to $V_{p r}$, we find

$$
\frac{d I_{p r}}{d V_{p r}}=-A_{c, e} e \int_{-e \Delta V}^{\infty} \dot{f}(E) d E
$$

Differentiating again

$$
\frac{d^{2} I_{p r}}{d V_{p r}^{2}}=-A_{c, e} e f(E)
$$

it is obviously, that the second derivatives of the probe current is proportional to the energy distribution. The maximum of $I^{\prime}\left(V_{p r}\right)$ and the zero point of $I^{\prime \prime}\left(V_{p r}\right)$ define the space potential (good agreement was found in using these techniques). 
Furthermore the density can be assessed by integrating the second derivative

$$
n_{e}=-\frac{1}{N A_{c, e} e} \int_{-\infty}^{V_{s p}} \frac{d^{2} I_{p r}}{d V_{p r}^{2}} d V_{p r},
$$

whereby $N$ is the normalization constant of the energy distribution function $f(E)$.

But unfortunately derivatives are frequently noisy, so that an accurate estimate of $n_{e}$ is often impossible. Hence a cubic spline routine [15] was applied to I(V) data.

It is important to remember that the derivations in the preceding paragraphs are only valid in the single probe model based on equation (2.34). These techniques are not applicable for the effects described in the following chapters.

If the electrons have a Maxwellian velocity distribution, the logarithm of the $I(V)$ function (2.34)

$$
\ln \left(-\frac{I+I_{i s}}{I_{e s}}\right)=\frac{e}{T_{e}} V_{p r}-\frac{e V_{s p}}{T_{e}}
$$

gives a straight line, where the slope can be used to estimate the electron temperature $T_{e}$. However the experimental data do not have a linear behavior between the electron current and voltage (fig 3.9). We are confident that the electrons have a Maxwellian distribution. Fitting the I(V) characteristic to a single probe model is thus not an appropriate way to determine the plasma parameters as density, temperature and space potential. 


\subsection{Asymmetric double probe model}

In the previous discussed single probe model the space potential was assumed to be constant for any probe potential. In the following considerations the entire probe configuration is assumed to be floating and the space potential will then be a function of applied voltage.

In order to drive higher current by increasing the probe voltage, according to equation (2.34), this current must be provided by the second electrode. Since the collection area of the limiter is finite and hence the collected ion current, it is impossible to draw such large current. Therefore the actual amount of current flowing through the probe is reduced and a electric field builds up to prevent higher current. This causes an increase of the space potential. The disturbance in the plasma potential was also observed in the floating potential along poloidal direction (fig 2.4) [16]. Although this is not a proof for the plasma potential disturbance, it is at least a indication that this may occur.

A higher space potential in the flux tube than in the surrounding plasma results in a poloidal electric field, which causes an $\vec{E} \times \vec{B}_{\theta}$ drift. This drift forces the particles to rotate around the flux tube. Since such a rotation without affecting other particles is physically not possible, a drag force lowers this rotation and spreads out the electric field. As a result, the flux tube is broadened toward the limiter (fig 2.5). This was first introduced by Guenther [17].

Consider both probes to be floating, but still drawing currents like 


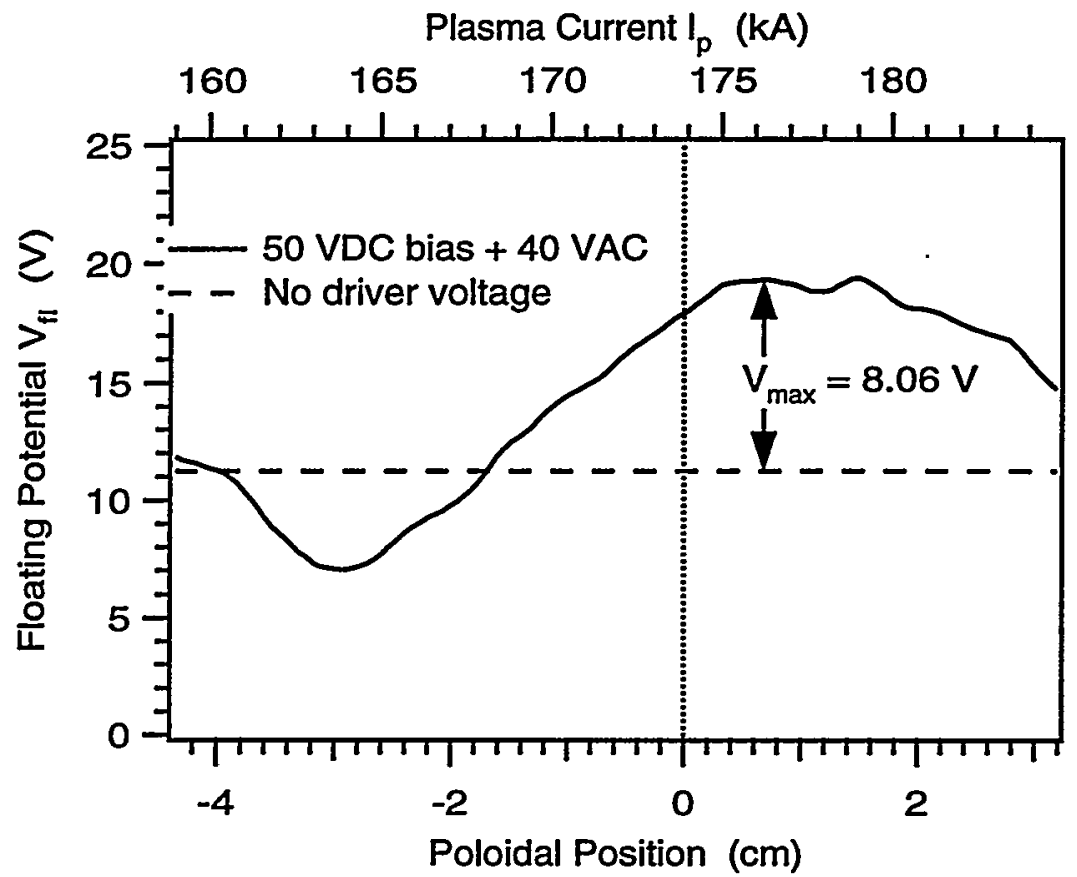

Figure 2.4: Floating Potential distribution in poloidal direction. An active probe causes growth of the space potential. 


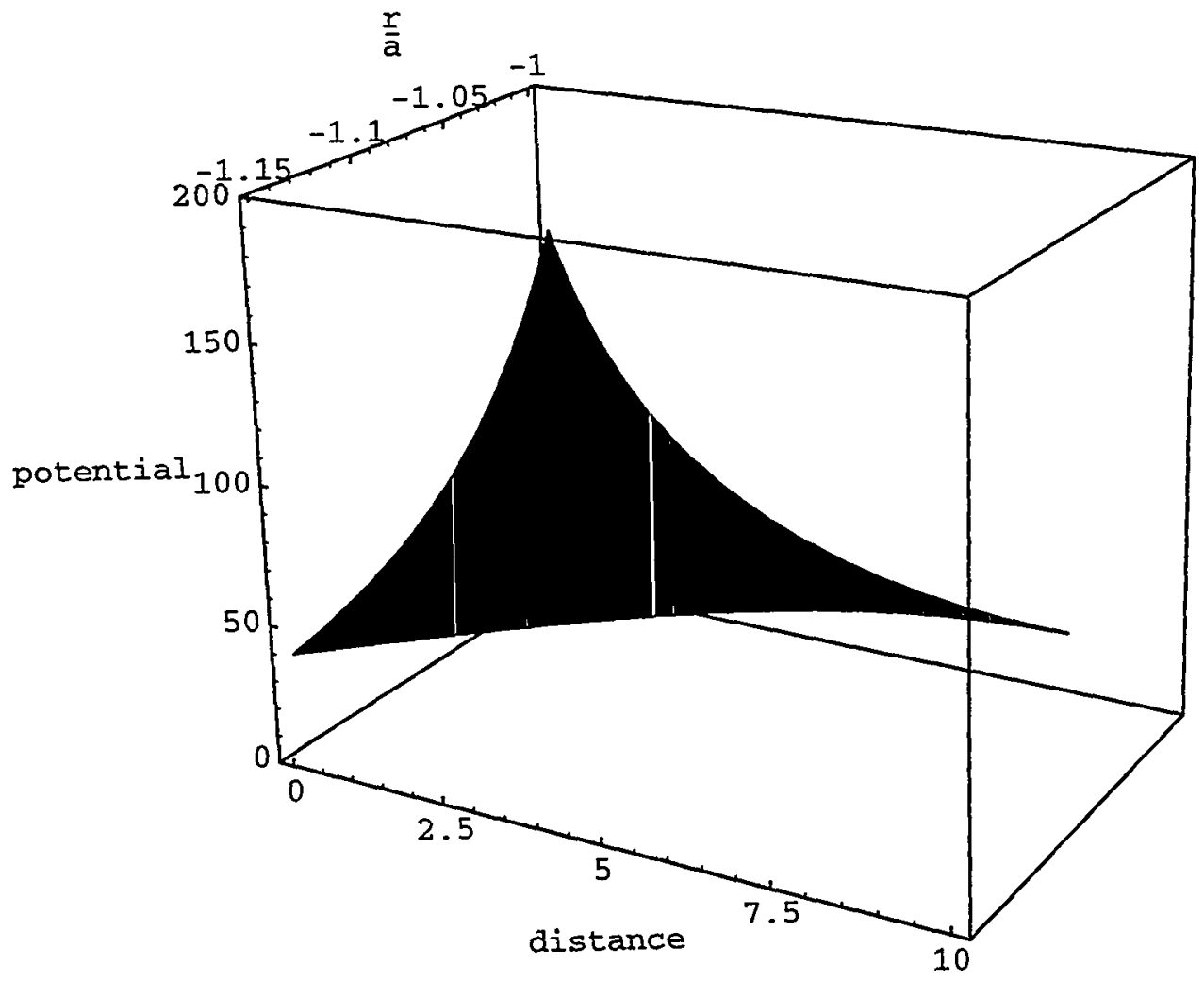

Figure 2.5: Space potential within the flux tube. The boundaries may not be correct. 
a single probe corresponding to eq. (2.34)

$$
\begin{aligned}
& I_{1}=-I_{i s}-I_{e s} \exp \left(\frac{e\left(V_{1}-V_{s p, 1}\right)}{T_{e}}\right) \\
& I_{2}=-I_{i s}-I_{e s} \exp \left(\frac{e\left(V_{2}-V_{s p, 2}\right)}{T_{e}}\right) .
\end{aligned}
$$

$V_{s p, 1}$ and $V_{s p, 2}$ are the space potential at each probe respectively. Due to the large size of the reference electrode (limiter), the collection area is more spatial expanded over several space potentials than that for the probe tip (see fig 2.5). The local space potentials at each probe are not necessary equal, at least not their spatial average. The difference is defined as floating potential. Consequently the voltage balance is

$$
V_{1}+V_{s p, 1}+V_{f l}=V_{2}+V_{p r}+V_{s p, 2}
$$

$\left(V_{p r}=V_{1}-V_{2}\right)$. Replacing $V_{s p, 2}$ in equation (2.49) and expressing $V_{s p, 1}$ by both equations gives

$$
V_{1}-\frac{T_{e}}{e} \ln \left(-\frac{I_{1}+I_{i s, 1}}{I_{e s, 1}}\right)=V_{2}-\frac{T_{e}}{e} \ln \left(-\frac{I_{2}+I_{i s, 2}}{I_{e s, 2}}\right) .
$$

Substitute the currents to the larger probe by using $I_{s, 2} \equiv \frac{1}{\alpha} I_{s, 1}$ and $I_{2} \equiv$ $-I_{1}$ and after rearranging one obtains

$$
\left(I_{1}+I_{i s, 1}\right) \frac{I_{e s, 1}}{\alpha} \exp \left(-\frac{e\left(V_{1}-V_{f l}\right)}{T_{e}}\right)=\left(-I_{1}+\frac{I_{i s, 1}}{\alpha}\right) I_{e s, 1} .
$$

Renaming the currents of probe 1 gives the asymmetric double probe characteristic

$$
I_{p r}=I_{i s} \frac{1-\exp \left(-\frac{e\left(V_{p r}-V_{f l}\right)}{T_{e}}\right)}{\alpha+\exp \left(-\frac{e\left(V_{p r}-V_{f l l}\right)}{T_{e}}\right)} .
$$

It is obviously that the $I_{p r}\left(V_{p r}\right)$ function no longer depends on the space potential. Only the ratio $\alpha$ of negative and positive saturation currents $\left(I_{-, s}, I_{+, s}\right)$, the temperature, ion saturation current (equivalent to 
the negative saturation currents) and now the floating potential are the parameters, which characterize the probe curve. The space potential increases always with the probe potential, so that this point will never be reached on the I-V profile. It seems that it is not possible to determine the space potential from such a characteristic. Indeed all techniques presented in the previous chapter are not relevant. But nevertheless, it is possible to derive an expression for the increasing effect of that potential.

Since the single probe equation (2.34) still describes the current, drawn by a particular probe with a potential $V_{k}$ different from the local space potential. we can equate the probe current (2.50) to the single probe current, (2.34)

$$
I_{i s} \frac{1-\exp \left(-\frac{e\left(V_{p r}-V_{f l}\right)}{T_{e}}\right)}{\alpha+\exp \left(-\frac{e\left(V_{p r}-V_{f l}\right)}{T_{e}}\right)}=-I_{i s}-I_{e s} \exp \left(\frac{e\left(V_{p r}-V_{s p)}\right.}{T_{e}}\right) .
$$

It is important to point out, that the ratio of electron and ion current is not $\alpha$, but, by defining a new constant $\beta$, it is

$$
\left|\frac{I_{e s}}{I_{i s}}\right|=\beta \text {. }
$$

$\beta$ is the theoretically calculated value for the ratio of the particle flux and their corresponding collecting areas,

$$
\begin{aligned}
\beta & \equiv \frac{\frac{1}{4} 4 r_{p r} l_{p r} \sqrt{\frac{8 T_{e}}{\pi m_{e}}}}{2 \pi r_{p r} l_{p r} \times 0.7 \sqrt{\frac{T_{e}}{m_{i}}}} \\
& =\frac{1}{0.7 \pi} \sqrt{\frac{2}{\pi}} \sqrt{\frac{m_{i}}{m_{e}}} .
\end{aligned}
$$

Table 2.1 shows $\beta$-values for different plasma ions.

The space potential expressed as a function of probe voltage is then

$$
V_{s p}=V_{p r}+\frac{T_{e}}{e} \ln \left[\beta \frac{\alpha+\exp \left(-\frac{e\left(V_{p r}-V_{f l}\right)}{T_{e}}\right)}{\alpha+1}\right] .
$$




\begin{tabular}{|ll||c|}
\hline & & $\beta$ \\
\hline \hline Hydrogen & ${ }_{1}^{1} \mathrm{H}$ & 15.55 \\
\hline Deuterium & ${ }_{1}^{2} \mathrm{D}$ & 21.98 \\
\hline Tritium & ${ }_{1}^{3} \mathrm{~T}$ & 26.93 \\
\hline Helium & ${ }_{2}^{3} \mathrm{He}$ & 26.90 \\
& ${ }_{2}^{4} \mathrm{He}$ & 30.99 \\
\hline
\end{tabular}

Table 2.1: $\beta$ values for various gases. The collection areas are assumed as in section (2.3.4).

Figure 2.6 verifies the accuracy of this equation: for large negative probe voltage only a slightly drop of the undisturbed potential occurs. On the contrary for large positive probe voltage an almost linear growth is observed. For $V_{p r}=0$ the undisturbed space potential is

$$
V_{s p}=\frac{T_{e}}{e} \ln \left[\beta \frac{\alpha+\exp \left(\frac{e V_{f l}}{T_{e}}\right)}{\alpha+1}\right] .
$$

If $\alpha$ is small, the equation (2.53) can be approximated as

$$
V_{s p} \approx \frac{T_{e}}{e} \ln \beta+\frac{T_{e}}{e} \ln \left[\exp \left(\frac{e V_{f l}}{T_{e}}\right)\right],
$$

what turns out after using the definition of $\beta$

$$
V_{s p}=\frac{T_{e}}{e} \ln \left(-\frac{I_{e s}}{I_{i s}}\right)+V_{f l},
$$

to be equal to equation (2.36) as derived in the single probe model. Also $\gamma$ and $\beta$ are related as $\gamma \equiv \ln \beta$.

If the collecting area of the reference electrode is sufficiently large that it can provide the current necessary to draw electron saturation cur- 
rent by the probe tip, the single probe model can be applied. This is equivalent to the case

$$
\left|\frac{I_{+, s}}{I_{-, s}}\right| \equiv \frac{A_{r e f}}{A_{p r}}>\frac{I_{e s}}{I_{i s}}
$$

Otherwise only the asymmetric double probe formulas give an accurate curve fit model. Another proof for this fact is, that equation (2.50) describes for $\alpha=0$ exactly the probe characteristic in the single probe model for $V_{p r}<V_{s p}$.

\subsection{Non-saturation effect of the ion current}

A flat ion saturation current is expected in most cases of Langmuir probes measurements. As shown in fig 3.13 , a significant non-saturation effect on the ion side was discovered. This is explained in general by a sheath growing with increasing negative probe voltages. Although the Debye length is small $(\lambda \approx 0.026 \mathrm{~mm})$, the sheath seems to be big enough to cause such effects.

In the following an estimate of the ion saturation current under the assumption of a strongly negatively biased probe is derived. If a probe repels electrons at large potentials, their density within the sheaths becomes almost zero. Then Poisson's equation is

$$
\nabla^{2}(\Delta V(\vec{x}))=-\frac{e}{\epsilon_{0}} n_{i}(\vec{x})
$$

$\left(\Delta V(\vec{x})=V(\vec{x})-V_{s p}\right)$ or in 1-dim coordinates and using $\Gamma_{i}=n_{i} v_{i}$

$$
\frac{d^{2}(\Delta V)}{d x^{2}}=-\frac{e}{\epsilon_{0}} \frac{\Gamma_{i}}{v_{i}} .
$$


Employing energy conversation to define $v_{i}$

$$
\frac{d^{2}(\Delta V)}{d x^{2}}=-\frac{e}{\epsilon_{0}} \Gamma_{i} \sqrt{\frac{-m_{i}}{2 e V}}
$$

which changes after multiplying by $d(\Delta V) / d x$ and integration to

$$
\begin{aligned}
&\left(\frac{d(\Delta V)}{d x}\right)^{2}=4 \Gamma_{i} \sqrt{\frac{m_{i} e}{2 \epsilon_{0}^{2}}}\left(\sqrt{-\Delta V}-\sqrt{V_{s p}-V_{0}}\right) \\
& \mathbb{1} \\
&\left(\sqrt{-\Delta V}-\sqrt{V_{s p}-V_{0}}\right)^{-\frac{1}{2}} d V=\frac{\sqrt[4]{8 m_{i} e \Gamma_{i}^{2}}}{\sqrt{\epsilon_{0}}} d x .
\end{aligned}
$$

This results by integration in

$$
-\frac{4}{3} \sqrt{(-\Delta V)^{\frac{3}{2}}+3 \Delta V \sqrt{V_{s p}-V_{0}}-4\left(V_{s p}-V_{0}\right)^{\frac{3}{2}}}=\frac{\sqrt[4]{8 m_{i} e \Gamma_{i}^{2}}}{\sqrt{\epsilon_{0}}}\left(x-x_{0}\right) .
$$

By taking the boundary condition at the sheath edge $\frac{d \Delta V}{d x}\left(x=x_{s h}\right)=V_{s h}$ the voltage dependence of the sheath thickness $d$ is expressed as

$$
d=\frac{4}{3} \frac{1}{\sqrt[4]{8 e T_{e}}} \sqrt{\frac{\epsilon_{0}}{0.7 n_{\infty}}} \sqrt{(-\Delta V)^{\frac{3}{2}}+3 \Delta V \sqrt{V_{s p}-V_{s h}}-4\left(V_{s p}-V_{s h}\right)^{\frac{3}{2}}},
$$

with the ion particle flux $\Gamma_{i}$ as in equation (2.30) .

The question comes up 'how to define the sheath edge potential ?'. It is usual to use the Bohm criterion $V_{s h}=V_{s p}-\frac{T_{\varepsilon}}{2 e}$, which leads to the final result

$$
d=\frac{4}{3} \frac{1}{\sqrt[4]{8 e T_{e}}} \sqrt{\frac{\epsilon_{0}}{0.7 n_{\infty}}} \sqrt{(-\Delta V)^{\frac{3}{2}}+3 \Delta V \sqrt{\frac{T_{e}}{2 e}}-4\left(\frac{T_{e}}{2 e}\right)^{\frac{3}{2}}} .
$$

But, since it is difficult in practice to fit such a function and the difference between the space and (such defined) sheath potential is small compare to the large negative probe bias (when the non-saturation effect becomes important), it is valid to set $V_{s h}=V_{s p}$. Thus

$$
d\left(V_{p r}\right)=\frac{4}{3} \frac{1}{\sqrt[4]{8 e T_{e}}} \sqrt{\frac{\epsilon_{0}}{0.7 n_{\infty}}}\left(V_{s p}-V_{p r}\right)^{\frac{3}{4}} .
$$


Thus the total collecting area for ions is extended by the fraction $\frac{d\left(V_{p} r\right)}{\tau_{p r}}$ and the ion current for ion attracting potentials is then

$$
I_{i}=I_{i s}\left(1+a\left(V_{s p}-V_{p r}\right)^{\frac{3}{4}}\right) \text {, }
$$

whereby

$$
a \equiv \frac{4}{3} \frac{1}{r_{p r} \sqrt[4]{8 e T_{e}}} \sqrt{\frac{\epsilon_{0}}{0.7 n_{\infty}}} .
$$




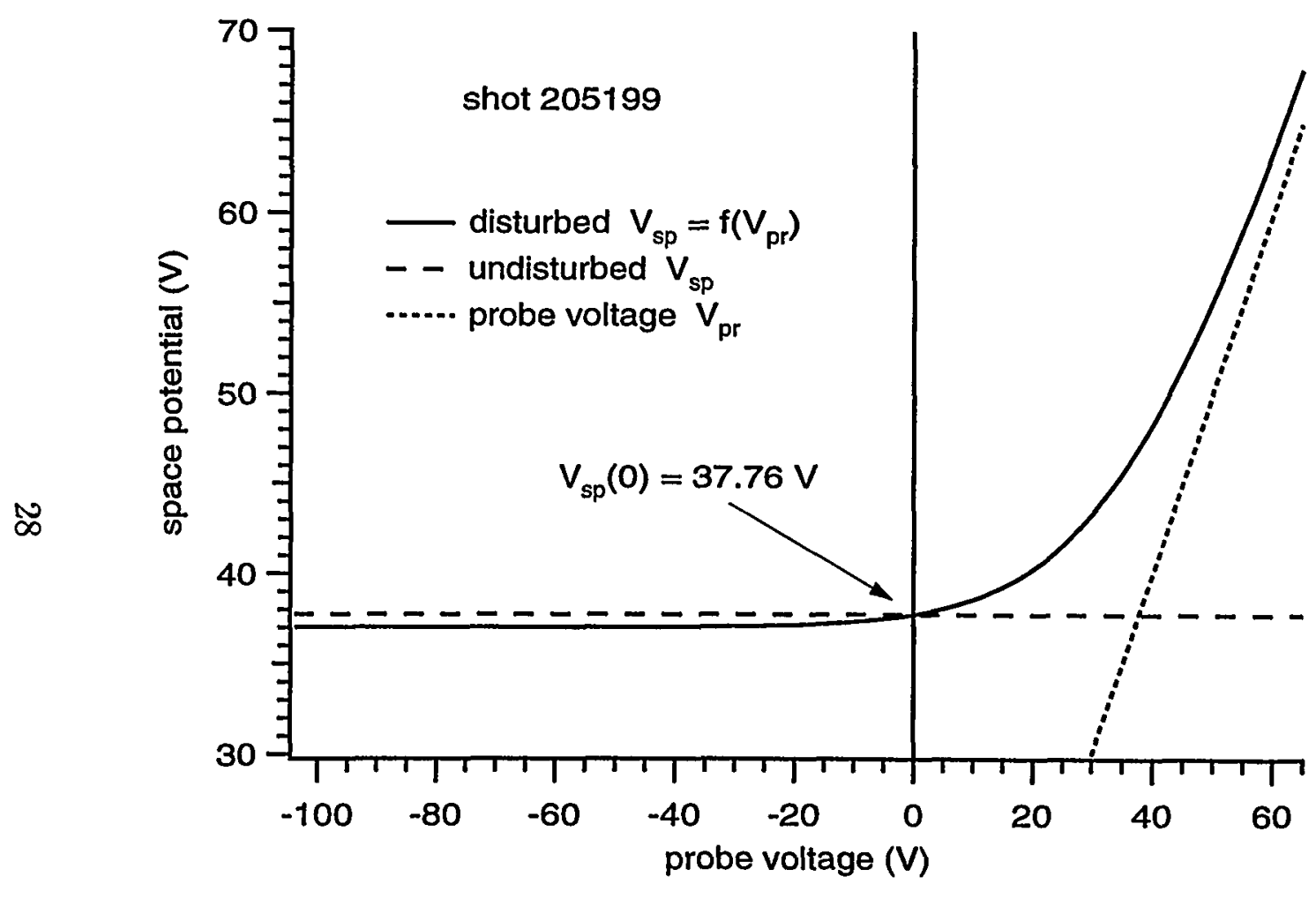

Figure 2.6: Space potential as a function of probe voltage. The plotted curve is based on density and temperature as in shot 205199 (see fig 3.6). 


\section{Chapter 3}

\section{Description of Experimental}

\section{Apparatus}

\subsection{Langmuir probes at the TEXT-U Toka- mak}

TEXT is a medium sized, ohmic heated Tokamak with a toroidal magnetic field $1.0 \leq B_{\theta} \leq 2.8 \mathrm{~T}$, plasma current $100 \leq I_{p} \leq 400 \mathrm{kA}$, chord average density $1 \times 10^{-19} \leq \bar{n}_{e} \leq 9 \times 10^{-19} \mathrm{~m}^{-3}$ and a major radius $R=1.0$ $\mathrm{m}$ [18]. Data were taken at conditions of a magnetic field $B_{\theta}=2.0 \mathrm{~T}$, a plasma current $I_{p}=200 \mathrm{kA}$ and a density $\bar{n}_{e}=2 \times 10^{-19} \mathrm{~m}^{-3}$. The limiters, positioned at a radius $a=27 \mathrm{~cm}$, define a circular plasma cross-section. Cylindrical Langmuir probes are used at TEXT. By using a reciprocator (pneumatic drive with a throw of $5 \mathrm{~cm}$ in approximately 40 millisecond) the probe head can operate in radial positions between 26 and $31 \mathrm{~cm}$.

The probe tips, each $0.5 \mathrm{~mm}$ in diameter and 2 millimeters in length, 


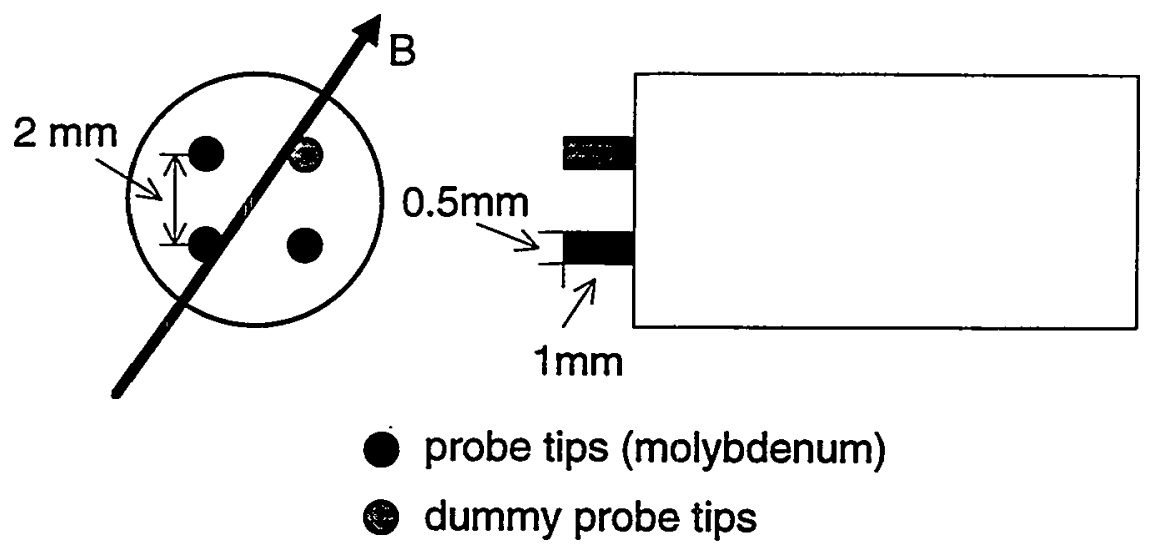

Figure 3.1: Schematic of probe head

were so arranged to the magnetic field, that probe shadowing was avoided (fig 3.1). One probe measured the floating potential, another one was biased by an AC-power supply.

\subsection{Power supply and data acquisition sys- tem}

In order to obtain a complete I-V characteristic the probe voltage was swept by a RF power source, which consists of a sine wavegenerator, power amplifier and an impedance matching transformer. The transformer in combination with a parallel capacitor is necessary to match the impedance required by the power amplifier. It is possible to sweep the voltage up to frequencies of $300 \mathrm{kHz}$. However, since no significant changes 
in the I-V curves at different frequencies were observed, the measurements focused on the frequency range $10 \leq f_{p r} \leq 50 \mathrm{kHz}$. Corrections for the cable capacitance $\left(\approx 3 \mathrm{~m}, C_{c}=275.6 \pm 6.3 \mathrm{pF}\right)$ were made during data analysis.

Both, the voltages of the biased probe and of the probe tip for floating potential comparisons, were monitored by a high-input-impedance isolator. A current probe combined with an amplifier (fig 3.2) measured the probe current. The data acquisition system is isolated from the power supply. Each output channel was stored, after digitizing with a 12 bit digitizer at a sampling frequency $f_{s}=5 \mathrm{MHz}$, in the TEXT-data system.

\subsection{Data analysis}

Every measured quantity is adulterated by noise and plasma fluctuations:

$$
I_{i}=I\left(V\left(t_{i}\right)\right)+\tilde{I}_{i}
$$

(note that $Q_{i} \equiv Q\left(t_{i}\right)$ are used interchangeable). The time average over a fluctuation must by definition be zero

$$
\left\langle\tilde{I}_{i}\right\rangle_{t} \equiv 0
$$

Therefore a boxcar averaging routine was applied to reduce current and voltage data to an appropriate I(V) curve. Although fluctuation levels are still accessible, a phase delay analysis is impossible, since for this a time domain analysis would be necessary.

After a hysterises was unexpectedly discovered, a correction for the 


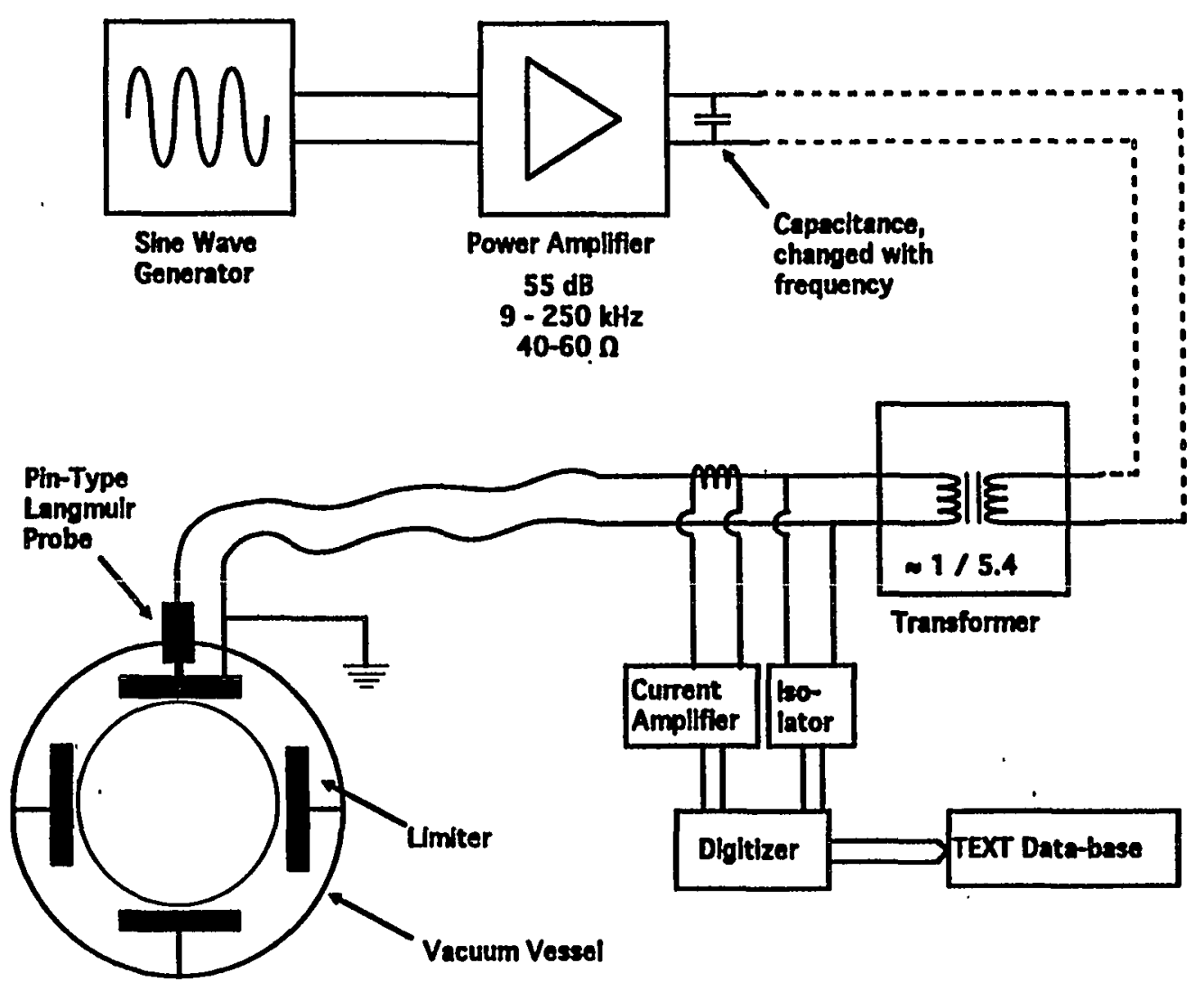

Figure 3.2: Electrical Circuit 
cable capacitance was applied. The current due to a capacitor is

$$
I(t)=\frac{1}{X_{C}} V(t)
$$

or as in the case of a AC-voltage

$$
I(t)=\frac{1}{i \omega_{p r} X_{C}} \dot{V}(t)
$$

whereby $X_{C}$ is the AC-impedance defined as

$$
X_{C}=\frac{1}{i \omega_{p r} C_{c}}
$$

with $\omega_{p r}=2 \pi f_{p r}$ as voltage frequency and $C_{c}$ as cable capacitance. Each data point is then corrected by the relation

$$
I_{i, \text { corr }}=I_{i}-C_{c} \dot{V}\left(t_{i}\right)
$$

A problem arises in calculating the time derivative of the voltage signal, since the sine wave was often deformed. However the usual approximation of three data points gives enough accuracy

$$
I_{i, \text { corr }}=I_{i}-\frac{1}{2} C_{c}\left(V_{i+1}-V_{i-1}\right) \text {. }
$$

This can explain the most part of the hysterises (fig 3.3). However it is still not understood why still a small hysterises remains at the frequency range of $50-200 \mathrm{kHz}$. Hysterises would be expected at higher frequency [19]. 


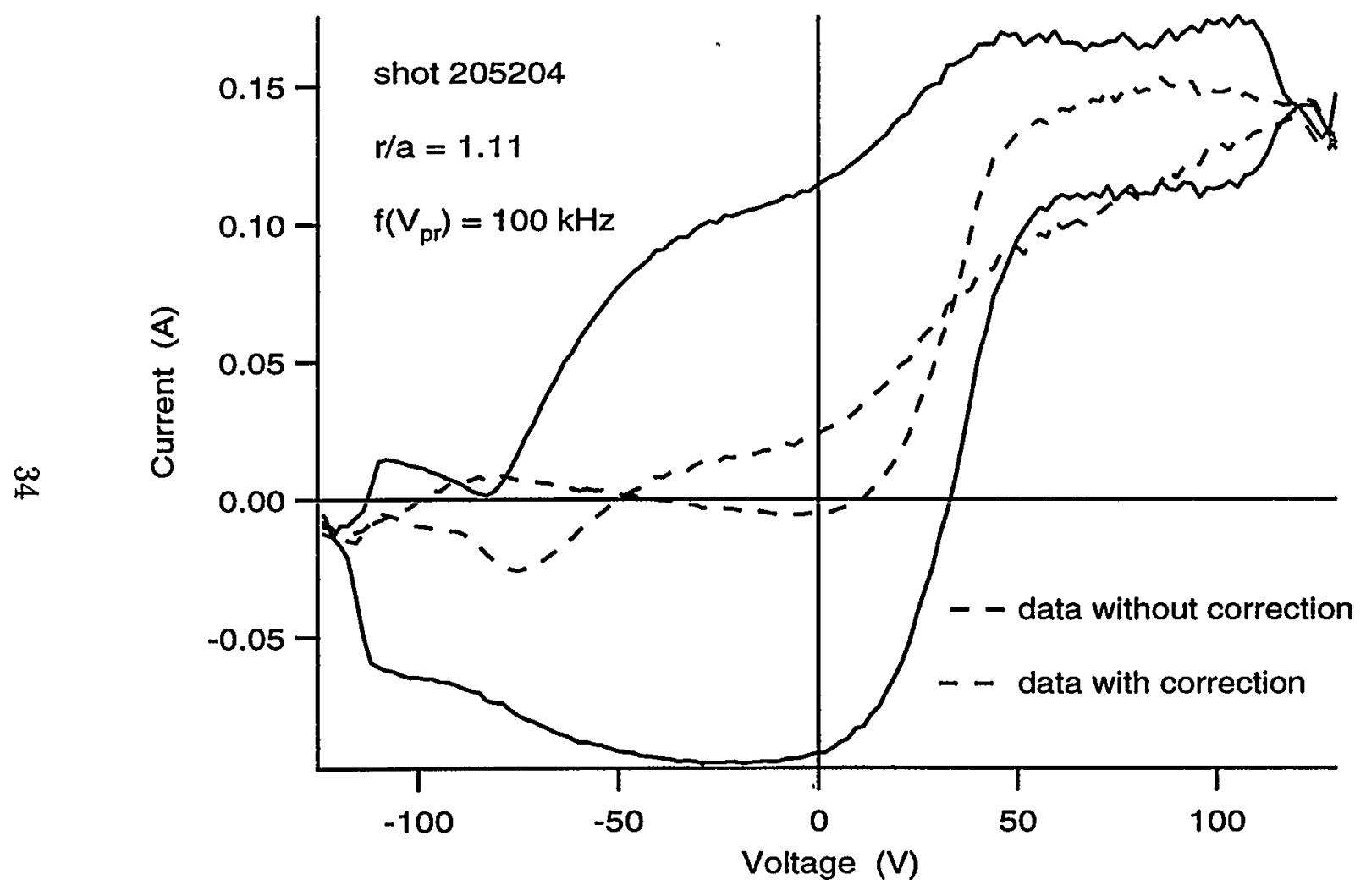

Figure 3.3: Comparison of boxcar-averaging with and without correction for the cable 


\subsection{Experimental results}

\subsubsection{Comparison of probe models}

Data were taken at four different radial position. After boxcaraveraging the I-V curves were obtained (fig 3.4). It is remarkable that the positive saturation current does not increase, although the density grows towards the plasma center. This is partially explained by the fact, that even the area of the reference electrode, which is not simple the projected area and rather complicated to calculate, might be changing with radius. Calculations of connection length did not indicate any large changes in connection length from the probe to a material surface. The ion saturation current shows the expected radial dependence. As mentioned in chapter (2.3.4), the positive saturation current can not be simply expressed by density and temperature. Not only the single probe model, but also the double probe model possesses an uncertainty for the positive saturation current. Hence only the ion saturation region is available for density measurements.

A single probe and a double probe model were applied to a particular shot. It is obvious from figure 3.5 that the single probe model does not follow the curve for probe potential $V_{p r}>V_{f l}$. The space potential for the numeric calculation of the single probe equation was determined by the first derivative plot (fig 3.7) and second derivative (fig 3.8).

The parameters as obtained by a least square fit for the double probe model (fig 3.6) do not clearly indicate that the single probe model is not applicable, since the ratio of positive and negative saturation currents 
$\left(\alpha^{-1}=21.77\right)$ is approximately the theoretical value of the electron and ion saturation ratio $(\beta=21.98$, table 2.1). But since a better fit is obtained by the double probe model, the single probe model seems not be valid.

Besides the logarithm plot (fig 3.9) of the shot shows clearly a nonlinear behaviour of the electron current respect to the voltage. This is in complete disagreement to equation (2.47). Since we believe that the electrons are Maxwellian distributed in the SOL and the equipartition time [20]

$$
\begin{aligned}
t_{e c} & =\frac{3 \sqrt{m_{e}}\left(4 \pi \epsilon_{0}\right)^{2}}{8 \sqrt{2 \pi} n e^{4} \ln \Lambda}\left(T_{1}+T_{2}\right)^{\frac{3}{2}} \\
& =6.4 \times 10^{-8} \mathrm{sec} \\
\Lambda & =\frac{3}{2} \frac{\left(4 \pi \epsilon_{0} T_{1}\right)^{3}}{e^{3} \sqrt{\pi n_{e}}}
\end{aligned}
$$

( $T_{1}$ was assumed to be $T_{1}=2 T_{2}=12 \mathrm{eV}$ ) is such small that a second electron distribution with a different temperature could be excluded, it must be stated out the single probe model as presented in chapter 2.4 is not a valid description of the probe characteristic in strong magnetic fields in the tokamak scrape off layer.

\subsubsection{Plasma parameter profiles}

A triple probe was used to compare the fitted plasma parameter density and temperature. A explaination of the triple probe technique, which has been successful upplied at TEXT for turbulence studies for years, can be found in [21].

The basic idea of the triple probe is to measure three points of a I-V characteristic. Assuming a single probe model, which includes a exponential behaviour of the current, gives the ion saturation current, floating 
potential and temperature. One probe tip is such dc-biased that it draws at negative voltage ion saturation current. The current returns through a identical probe tip and the probe potential $V_{+}$of the second probe is measured. An independent third probe tip gives the floating potential.

Apparently the triple probe technique gives higher values for density and temperature than the single probe tip (fig 3.10 and 3.11). This is mainly caused by the fact, that the triple probe model is based on the single probe model. In addition it neglects the effect of non-saturation of the ion current. This results in higher $I_{i s}$-values and hence in high values for the densities.

The calculated values of space potential (fig 3.12), based on the double probe model are in good correlation with equation (2.37).

\subsubsection{Non-saturation effect}

A magnification of the $I(V)$ curve (fig 3.13) in the ion saturation region indicates a significant non-saturation effect (fig 3.13). This was also found in double probe (two identical probe tips of comparable size to the probe tip used for the single probe measurements) measurements (fig 3.14) [22].

A fit routine using the ion saturation current $I_{i s}$, multiplier $a$ and power $p$ as free parameter

$$
I_{p r}\left(V_{p r}\right)=I_{i s}\left(1+a\left(V_{s p}-V_{p r}\right)^{p}\right)
$$

was applied to the data in the ion saturation region. In table 3.1 the obtained fit values are compared with the theoretical calculated values for a, given by (2.58). The rough agreement is a indication that the sheath 


\begin{tabular}{|l|l|l|ll|ll|}
\hline shot & $r / a$ & $a_{\text {theo }}$ & \multicolumn{2}{|c|}{$a_{\text {exp }}$} & \multicolumn{2}{|c|}{$p$} \\
\hline \hline 205199 & 1.11 & 0.017 & 0.0080 & \pm 0.018 & 0.99 & \pm 0.375 \\
\hline 205202 & 1.11 & 0.015 & 0.080 & \pm 0.010 & 0.62 & \pm 0.04 \\
\hline 205203 & 1.11 & 0.015 & 0.014 & \pm 0.135 & 0.95 & \pm 1.49 \\
\hline 205214 & 1.07 & 0.014 & 0.016 & \pm 0.055 & 0.97 & \pm 0.44 \\
\hline 205215 & 1.07 & 0.015 & 0.012 & \pm 0.042 & 0.93 & \pm 0.50 \\
\hline 205216 & 1.07 & 0.014 & 0.13 & \pm 0.11 & 1.1 & \pm 0.8 \\
\hline 205224 & 1.04 & 0.0066 & 0.0061 & \pm 0.0148 & 0.91 & \pm 0.33 \\
\hline 205225 & 1.04 & 0.0073 & 0.0078 & \pm 0.034 & 0.96 & \pm 0.60 \\
\hline
\end{tabular}

Table 3.1: Comparison of theoretical and experimental values for non-saturation effect.

growth with increasing negative voltage can explain the non saturation of the ion current.

An additional experiment (one shot only) with a larger probe $\left(r_{p r}=\right.$ $1.3 \mathrm{~mm}, l_{p r}=12 \mathrm{~mm}$ ) however didn't show a dramatic non-saturation in ion current (fig 3.15). Besides according to the theory, the non-saturation should vanish for very large probes. In addition the probe length was of order of the radial scale for the plasma density and temperature.

But nevertheless the formulae presented in chapter 2.6 can be considered only as an approximate explanation. Especially, since the space potential was treated as constant, which is only true for large negative potential (fig 2.6). Of course it would be desirable to fit the complete probe characteristic including this effect. The double probe equation would than 
change to

$$
I_{p r}=I_{i s}\left(1+a\left(V_{s p}\left(V_{p r}\right)-V_{p r}\right)^{p}\right) \frac{1-\exp \left(-\frac{e\left(V_{p r}-V_{f l}\right)}{T_{e}}\right)}{\alpha+\exp \left(-\frac{e\left(V_{p r}-V_{f l}\right)}{T_{e}}\right)}
$$

( $V_{s p}$ as in equation (2.52)) Since it is difficult to do a least square fit with such a function, it seems reasonable to combine the space potential function (2.52) and the single probe equation (2.38) as fitting function. But unfortunately even a modified IDL $^{\mathrm{TM}}$ routine didn't give satisfying results. 


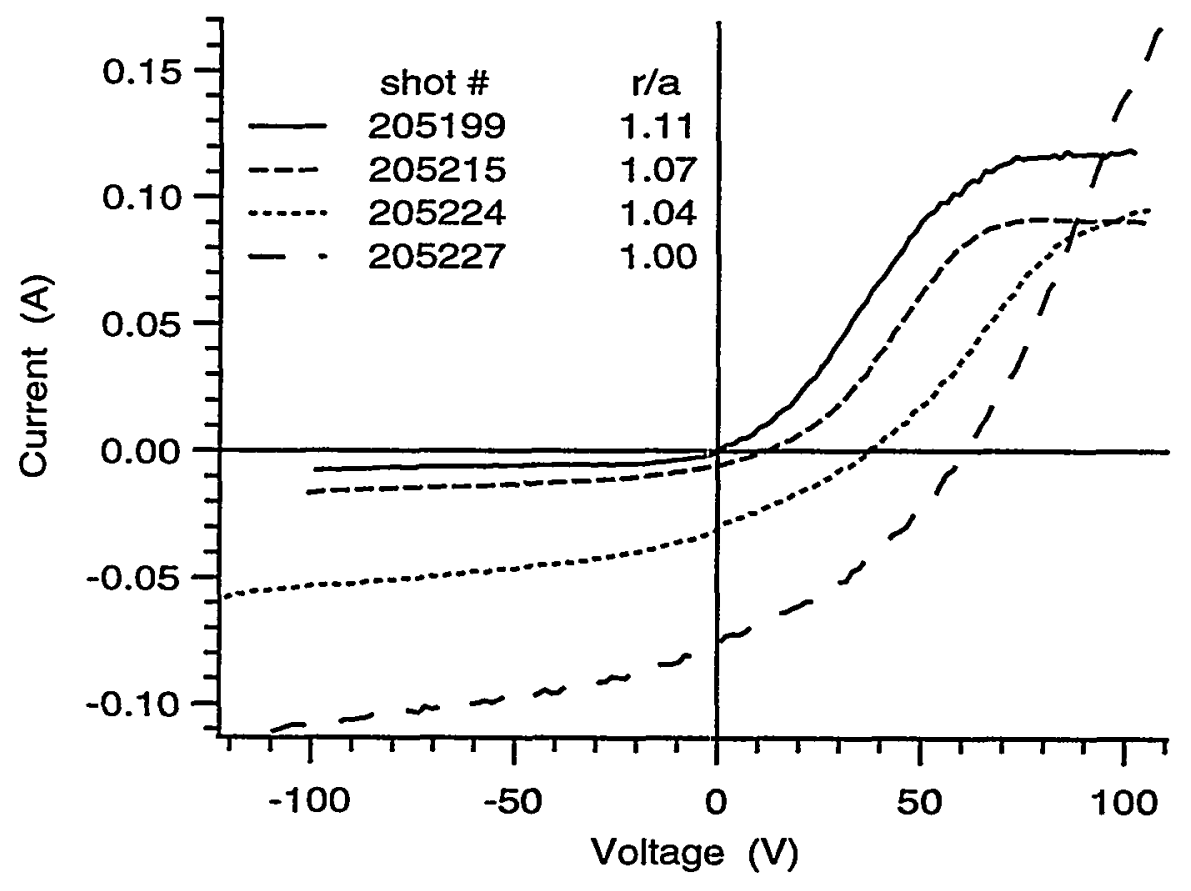

Figure 3.4: I-V characteristics of several shots at same discharge conditions, but different radial positions. 


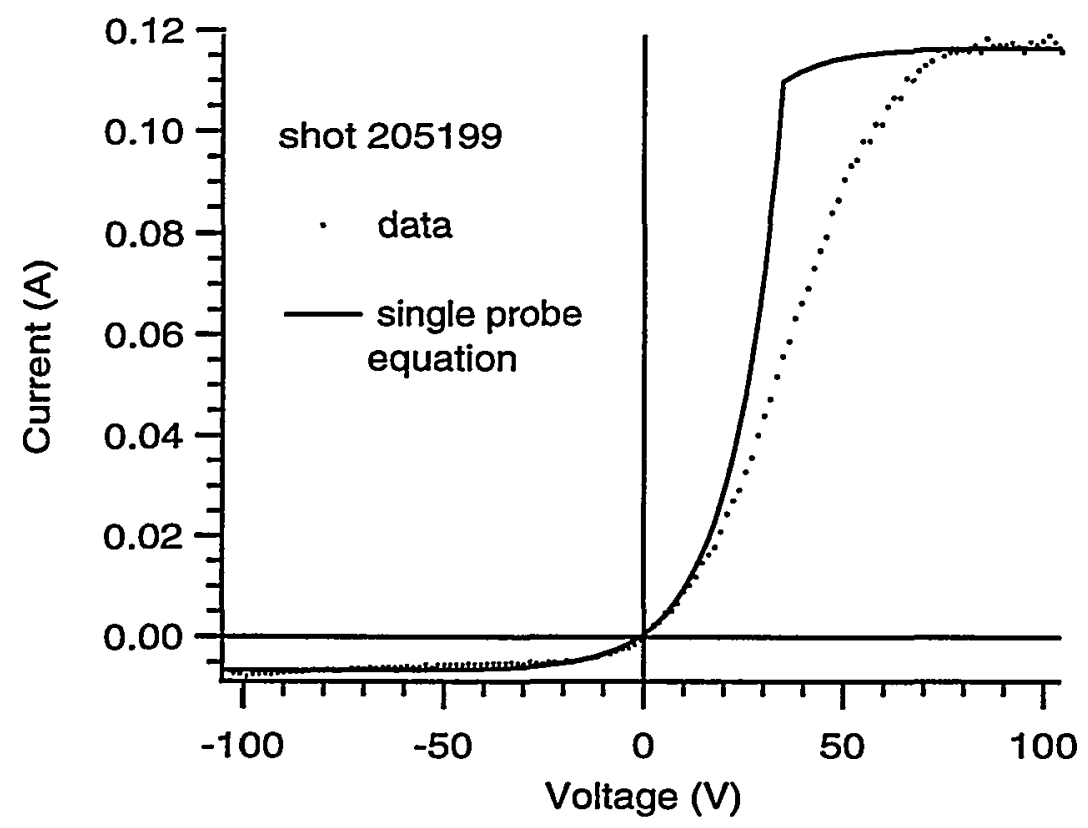

Figure 3.5: Comparison of data to the corresponding curve of a single probe model as in equation (2.41).

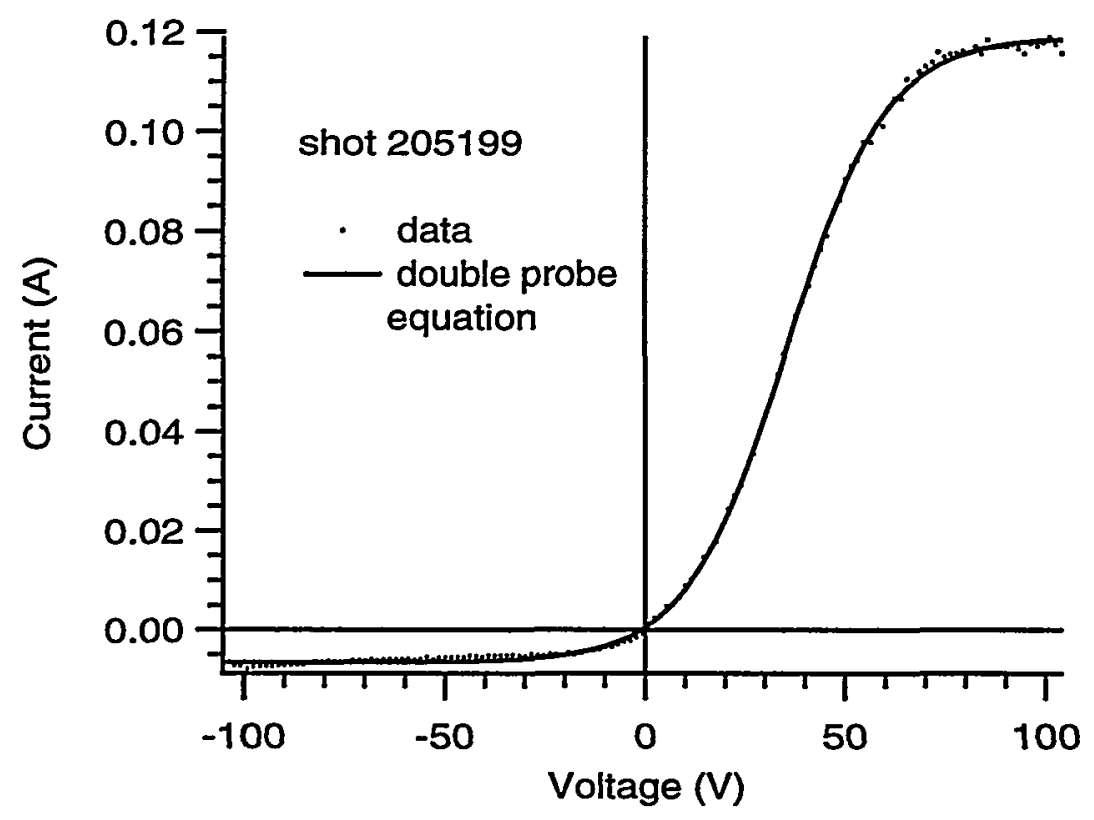

Figure 3.6: Curvefit of same data as above based on the asymmetric double probe equation (2.50). 


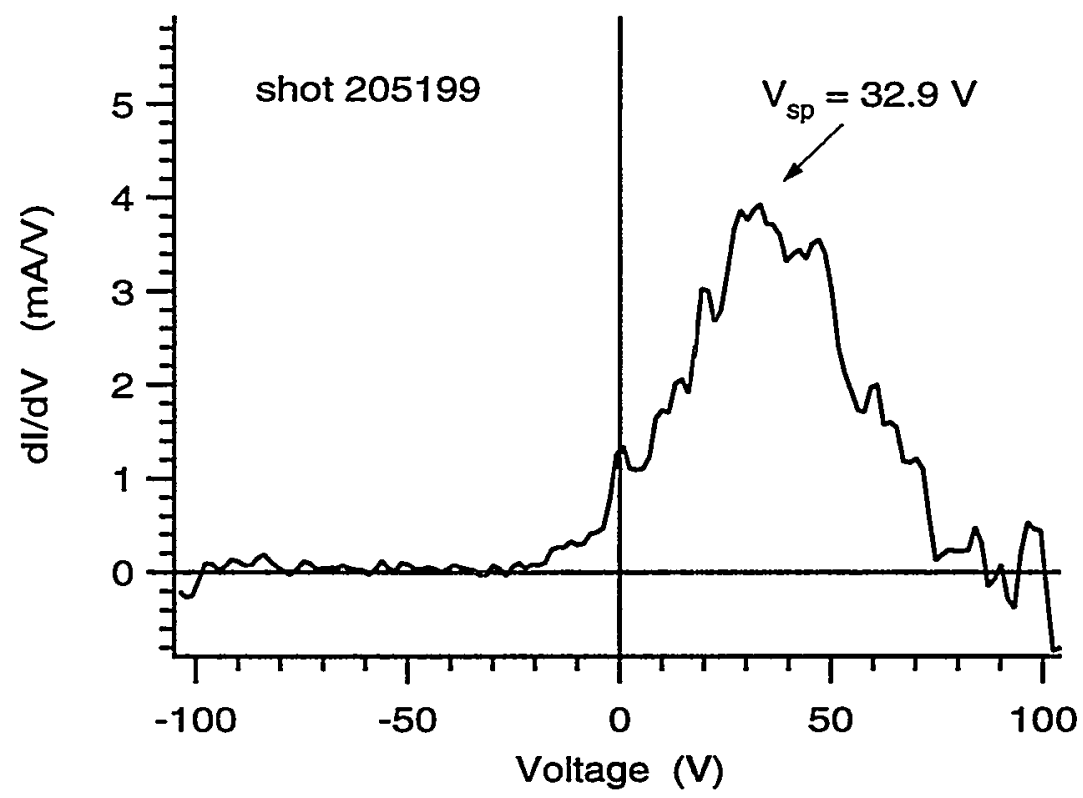

Figure 3.7: First derivative of $\mathrm{I}(\mathrm{V})$ curve of a probe tip positioned $3 \mathrm{~cm}$ behind the limiter.

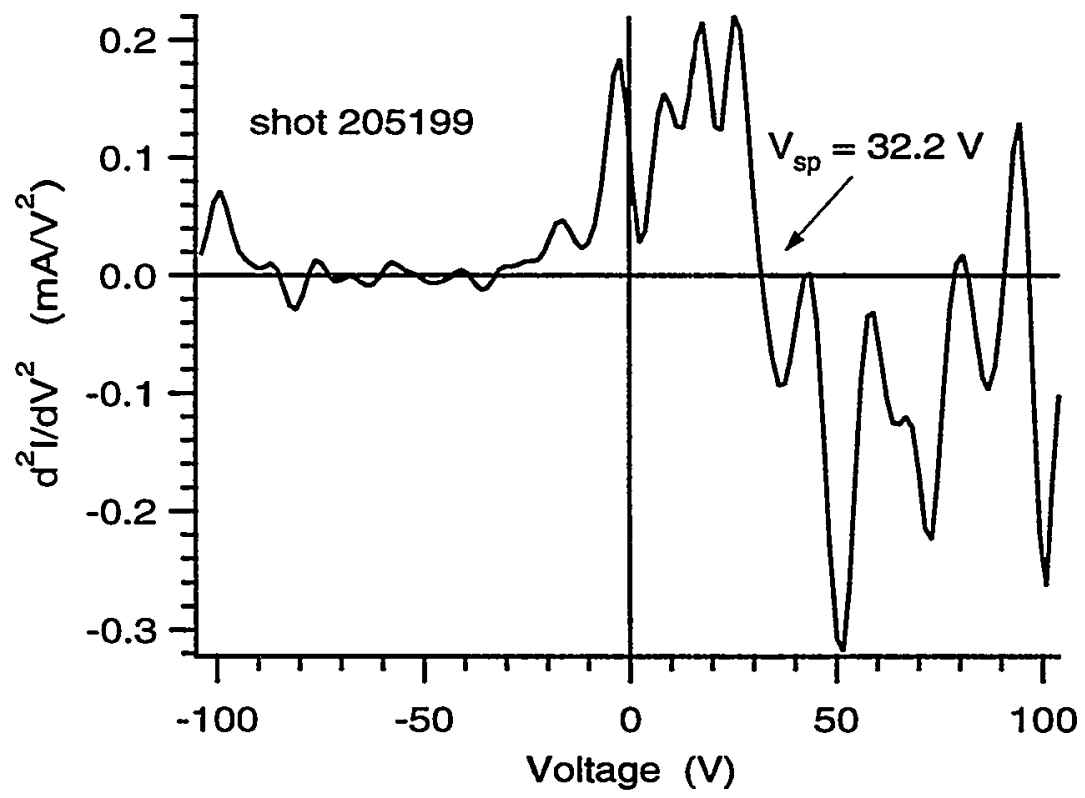

Figure 3.8: Second deriveative, after applying a cubic spline routine to the first deivative. 


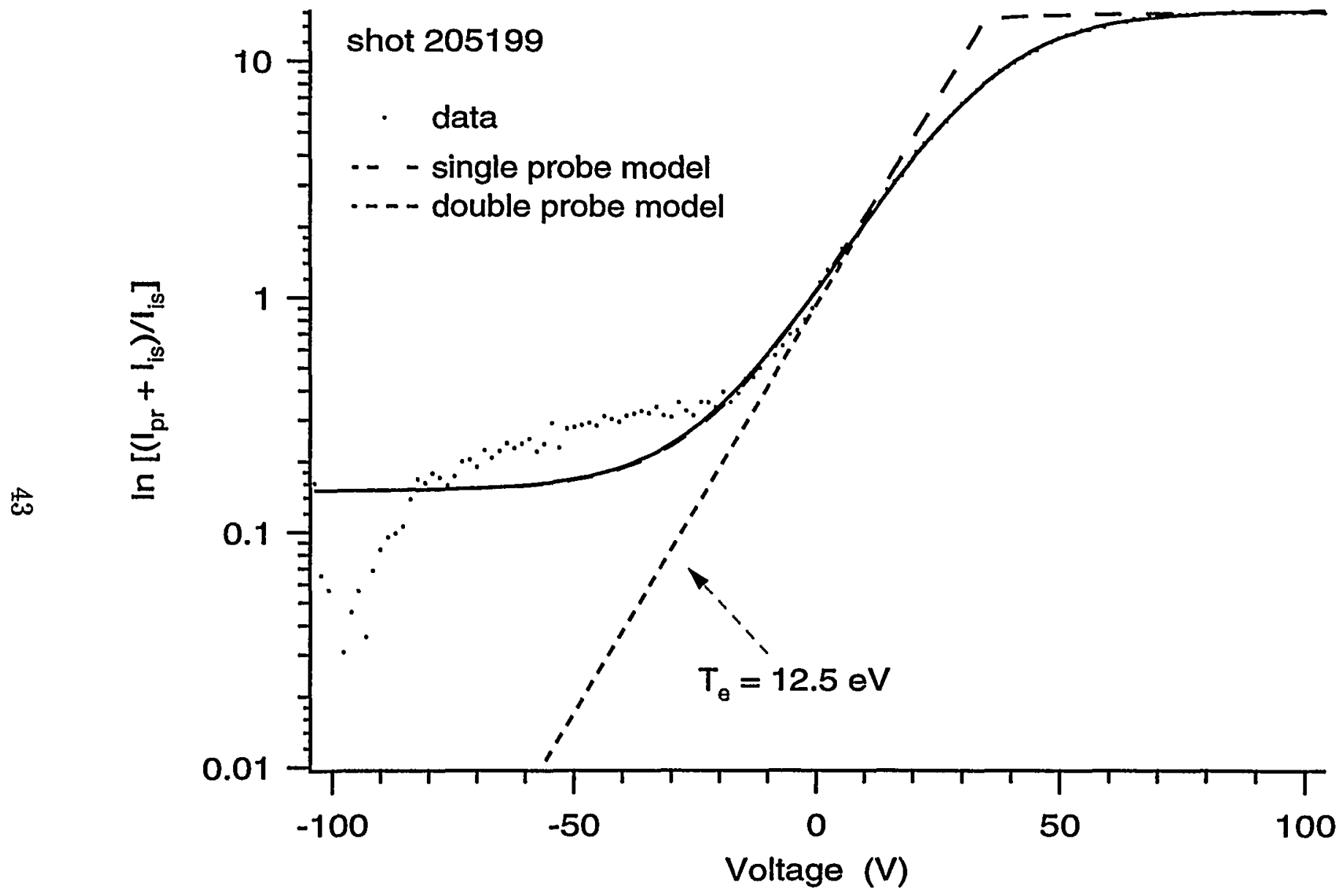

Figure 3.9: Logarithm Plot after subtracting the ion-saturation current. Additionally the logarithm plots of the curves as in figure 3.5 and 3.6 are shown. 


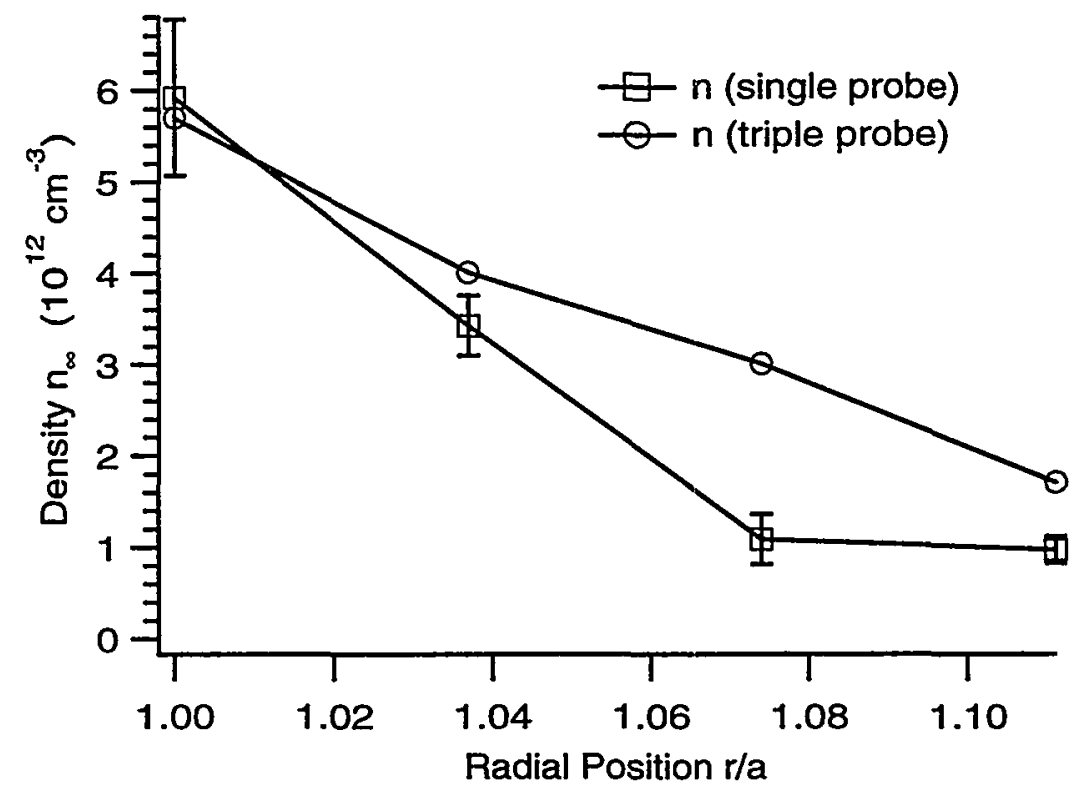

Figure 3.10: Density Profiles obtained by curve-fitting using the double probe model and averaging over several shots at same positions respectively. 


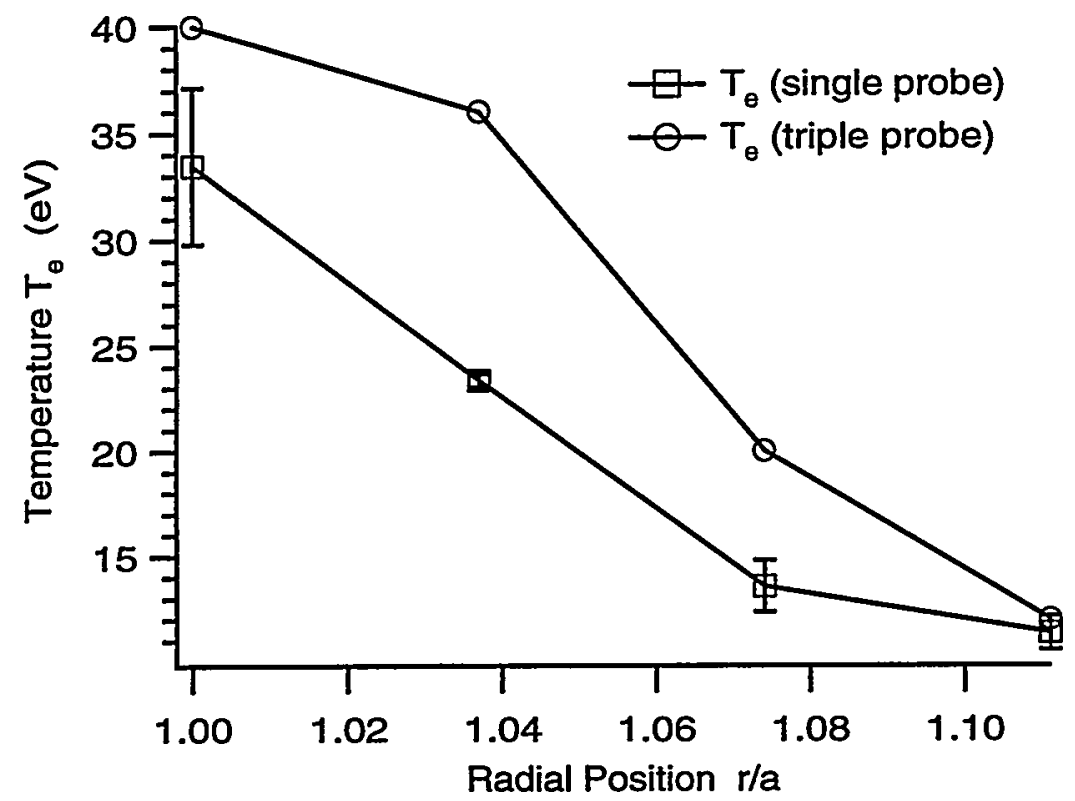

Figure 3.11: Temperature Profile based on same shots as in figure 3.10. 


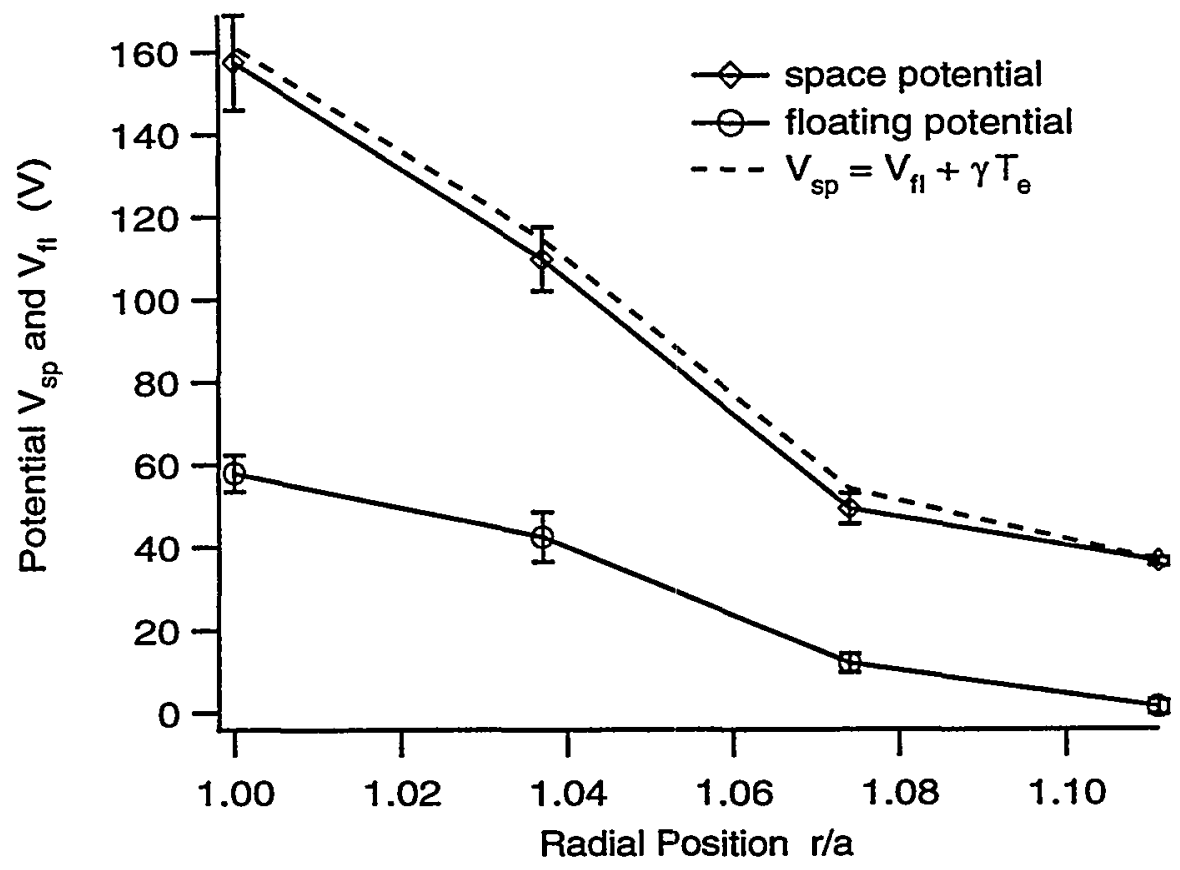

Figure 3.12: Profile of space and floating potential. 

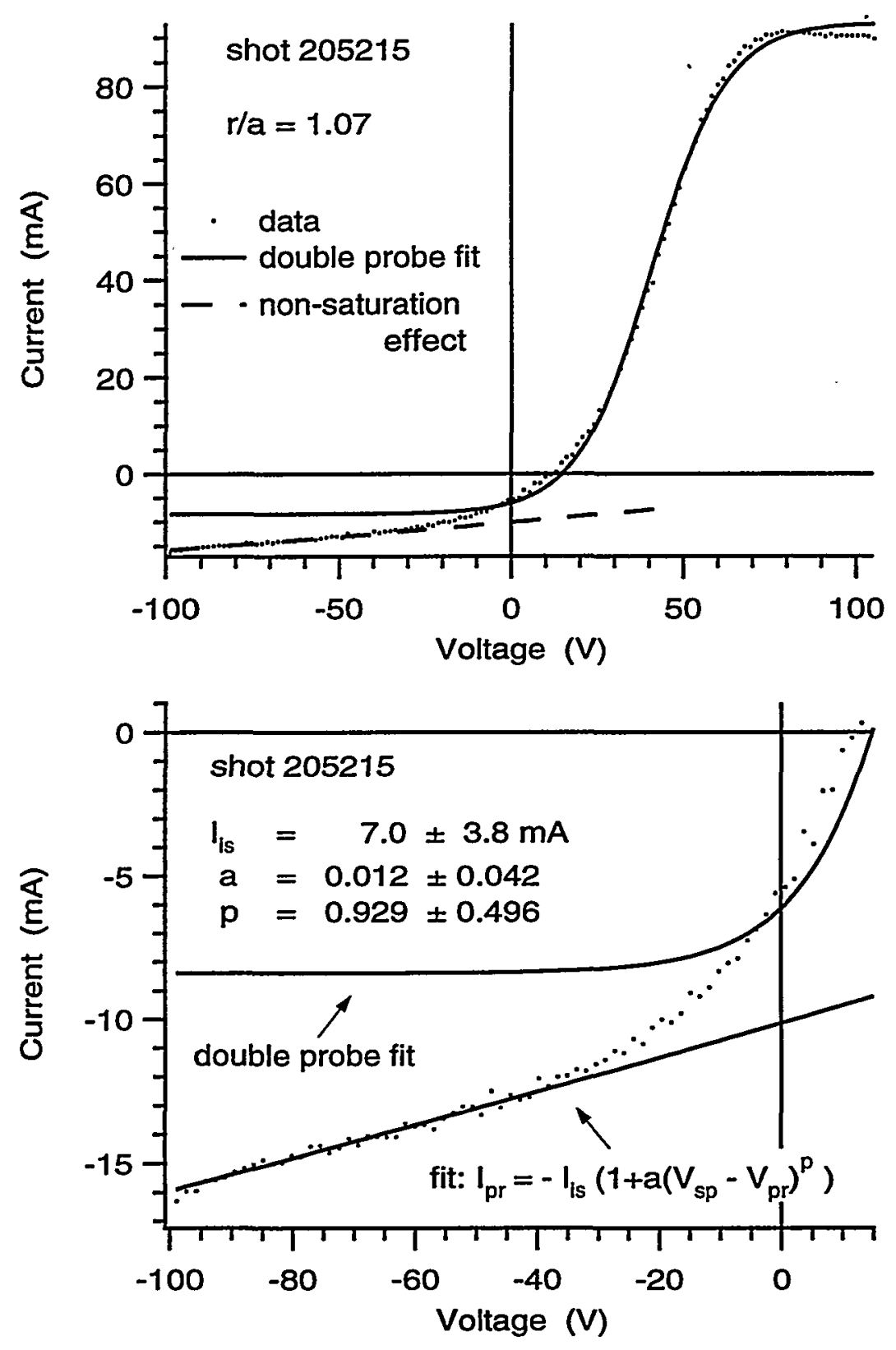

Figure 3.13: Discharge with probe tip $2 \mathrm{~cm}$ behind the limiter. The solid curve shows the results of a regular asummetric double probe fit. 


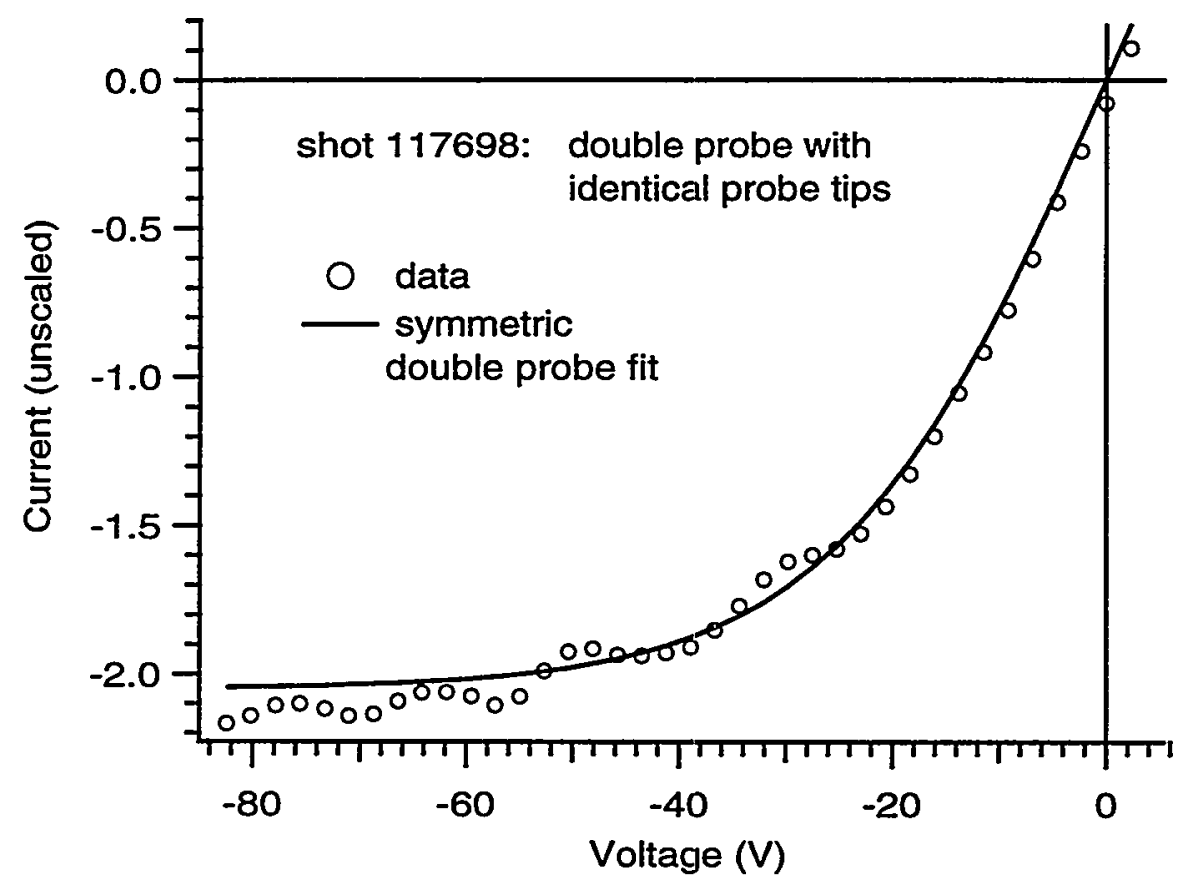

Figure 3.14: Data of a symmetric double probe configuration used for turbulence study at TEXT before the upgrade. 


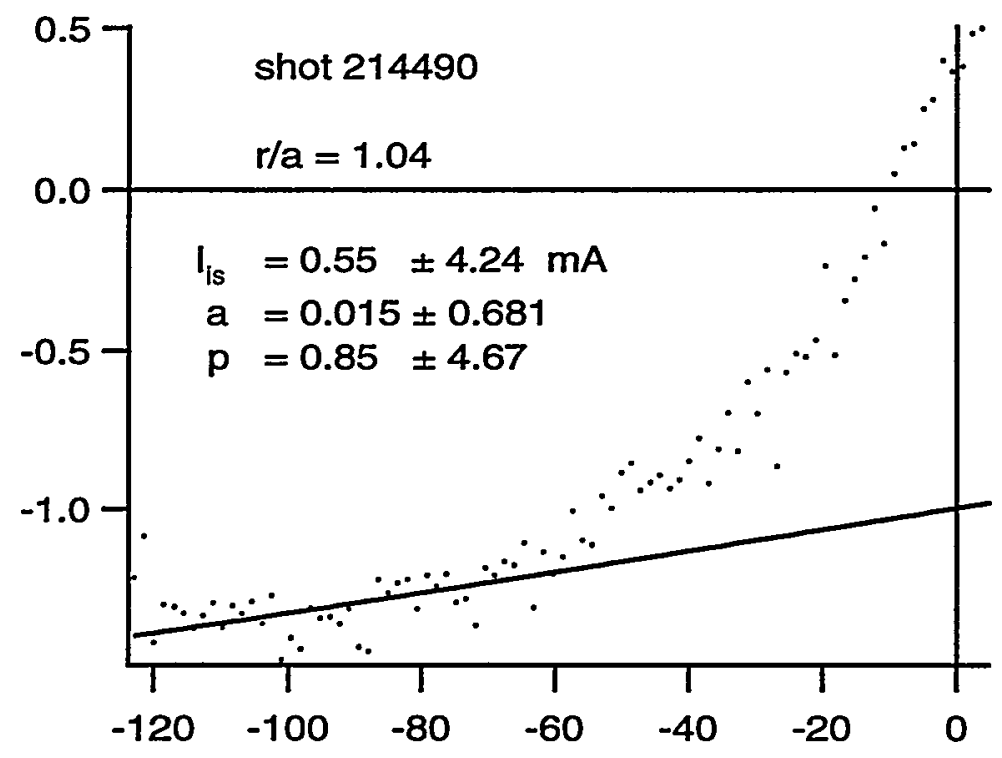

Figure 3.15: Single probe measurements with a large probe tip. The probe was DC-biased superimposed by a AC-voltage. 


\section{Chapter 4}

\section{Conclusion}

Two probe models to determine the space potential, plasma density and temperature in the scrape off plasma layer were presented. The main different attribute is the space potential. In the single probe model it is assumed to remain constant for all probe voltages. On the contrary in the double probe model it is considered to be increasing with probe potential. Hence the probe will never reach the electron saturation level. The term positive saturation current should be rather used for this part of the I(V) characteristic than electron collection current. The term electron collection current has meaning only in the single probe model.

The typical parameter to characterize which probe model should be employed for the data acquisition is the ratio of the two saturation levels. Since this ratio is proportional to the ratio of the collection areas of the probe tip and the reference electrode (in most cases the limiters), the use of the double probe model is always advised if this ratio is smaller than the predicted electron and ion saturation current ratio. If the area of the 
larger probe is so large that the small probe can collect electrons, the single probe model is the best describtion.

The probe tip used for this experiment was still too large to obtain a real single probe $\mathrm{I}(\mathrm{V})$ characteristic. An experiment should be carried out either by using a smaller probe tip, or by increasing the magnetic field such that the electron collection current drops sufficiently down. Since the electron particle flux is connected to a perpendicular flux tube, measurements with a sufficiently small enough probe tip in discharges with different magnetic fields could give a scaling for the perpendicular diffusion coefficient $D_{\perp}$. But nevertheless a calibration of the particle flux equations should be done along with measurements of the density and temperature by far infrared interferometry (FIR) and Thompson scattering measurements.

Although it is impossible to determine the space potential directly from the probe characteristic, the probe voltage dependence of the space potential could be derived. Thus the space potential is still available. However, no other diagnostic technique measures space potential directly except HIBP. Although the accuracy of this diagnostic may be not high enough in the SOL to serve as comparison to the Langmuir probe, it would test the asymmetric double probe theory.

Of course the question remains how to demonstrate the grow of the space potential in the flux tube. The measurement of the floating potential at different poloidal positions (either by sweeping the plasma current to move the magnetic field lines or by using a multiple linear probe tip array) can show how far the space potential is disturbed by the probe potential [16]. Another way to investigate this is a probe configuration consisting of a large probe tip positivly DC-biased with respect to the limiter and 
a second - very small - probe tip, positioned on the same field line for single probe measurements.

Besides the new interpretation of Langmuir probe characteristics, unexpected effects as a non-saturation of the ion current and a hysterises, unmistakable due to the plasma, were discovered. Since the triple probe technique measures only three points of the I(V)-curve, it is impossible to justify the model used by the triple probe. The obtained values may therefor be slightly adulterated. In addition there might be problems with probe shadowing, which the single probe clearly avoids. But still triple probes are the only technique for turbulence study, even if the absolute values may be in error.

Of course the investigation of the non-saturated ion current is not complete. But nevertheless the explanation given in chapter (2.6) is a start for further resarch in this matter. The same is true as for the "space potential": measurements with even smaller probes could increase this effect. Certainly a cooling of the probe would help using them even close to the limiters.

Even after seventy years of employement of Langmuir probes as plasma diagnostic technique, the theory is not understood. But there are also several features, which can be predicted accurately. Once the modeling of the density, temperature and potentials is verfied, the single probe technique is a reliable diagnostic for plasma profile measurements. 


\section{Bibliography}

[1] Industry statistics, The Oil and Gas Journal, December Issues 1951 thru 1992.

[2] M.A. Meier, Langmuir Probe Investigation of the Effect of Electron Cyclotron Resonance Heating on the Edge Plasma of the Texas Experimental Tokamak, Thesis, The University of Texas at Austin, (August 1992).

[3] H.M. Mott-Smith, I. Langmuir, "The Theory of Collectors in Gaseous Discharges", Physical Review, V 28, (October 1926).

[4] F.F. Chen, "Electric Probes", in Plasma Diagnostic Techniques, edited by R.H. Huddlestone and S.L. Leonard, Academic Press, New York 1965.

[5] J.G. Laframboise, Theory of Spherical and Cylindrical Langmuir Probes in a Collisionless, Maxwellian Plasma at Rest, UTIAS Report No.100, (1966).

[6] P.C. Stangeby, "Effect of Bias on Trapping Probes and Bolometers for Tokamak Edge Diagnosis", Journal of Physics D: Applied Physics, V 15, (1982). 
[7] I.H. Hutchinson, Principles of Plasma Diagnostics, Cambridge University Press, (1987).

[8] P.C. Stangeby, "Plasma Sheath Transmission Factors for Tokamak Edge Plasmas", The Physics of Fluids, V 27, No.3, (March 1984).

[9] I.H. Hutchinson, "A Fluid Theory of Ion Collection by Probes in Strong Magnetic Fields with Plasma Flow", The Physics of Fluids, V 30, No.12, (December 1987).

[10] P.C. Stangeby, "Comments on 'A Fluid Theory of Ion Collection by Probes in Strong Magnetic Fields with Plasma Flow'[Phys.Fluids 30, 3777 (1987)]", The Physics of Fluids, V 31, No.9, (September 1988).

[11] I.H. Hutchinson, "Reply to the Comments of Stangeby", The Physics of Fluids, V 31, No.9, (September 1988).

[12] R.D. Bengtson, J.Boede, W.L. Rowan, "Ion Temperature Profile Measurements Using the Far Line Wings of $\mathrm{H}_{\alpha}$ ", Review of Scientific Instruments, V 57, No.8, (August 1986).

[13] J.G. Laframboise, J. Rubinstein, "Theory of a Cylindrical Probe in a Collisionless Magnetoplasma", The Physics of Fluids, V 19, No.12, (December 1976).

[14] J.R. Sanmartin, "Theory of a Probe in a Strong Magnetic Field", The Physics of Fluids, V 13, No.1, (January 1970).

[15] A. Savitzky, M.J.E. Golay, "Smoothing and Differentiation of Data by Simplified Least Squares Procedures", Analytical Chemistry, V 36, No.8, (July 1964). 
[16] B. Richards, T. Uckan, et al., "Edge Diagnostics and Feedback Control Using an Active Probe on TEXT", 21st EPS Conference on Controlled Fusion and PLasma Physics, Contributed Papers, V 18B, Part 1, (June 1994).

[17] K. Guenther, A. Herrmann, M. Laux, P.Pech, H.-D. Reiner, "Characteristics of electric probes in the tokamak SOL: Influences of the magnetic field", Journal of Nuclear Materials, V 176 \& 177, 1990.

[18] K.W. Gentle, Nucl.Technol. Fusion 1 (1982) 479.

[19] P. Verplancke, "Changes of the Langmuir characteristic at frequencies up to ion cyclotron resonance", Seminar, presented at the University of Texas at Austin, (October 1994).

[20] L. Spitzer, Physics of fully ionized gases, Interscience Publisher, New York 1962.

[21] M. Kamitsuma, Sin-Li Chen, Jen-Shih Chang, "The Theory of the Instantaneous Triple-Probe Method for Direct of Plasma Parameters in Low-Density Collisionless Plasma", Journal of Physics D: Applied Physics, 10, (1977).

[22] H. Lin, Turbulence and Transport Studies in the Edge Plasma of the TEXT Tokamak, Dissertation, The University of Texas at Austin, (October 1991). 
VITA

Stefan Jachmich

After receiving the Abitur degree from the Alexandervon-Humboldt Gymnasium, Schweinfurt, Germany, in 1990, he entered the Julius-Maximillian Universität, Würzburg, Germany. In summer 1992 he received the Vordiplom degree in physics at this university. After one year of graduate study at the same university he entered in Fall 1993 The Graduate School of the University of Texas at Austin.

Permanent address:

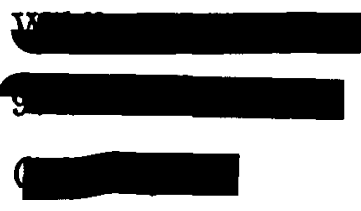

This thesis was typed by the author and prepared with $\mathrm{IAT}_{\mathrm{E}} \mathrm{X}, \mathrm{IGOR}$, and PowerDraw. 